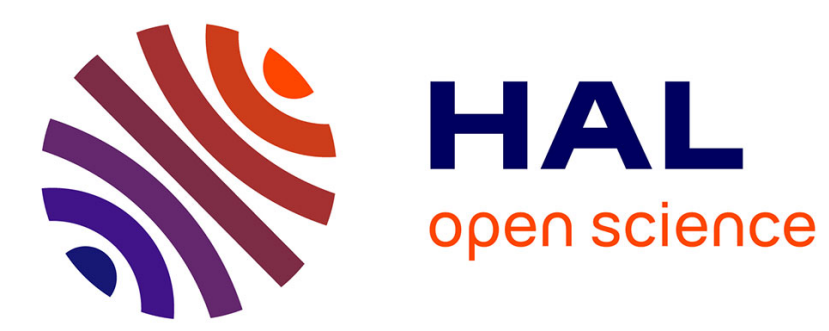

\title{
Advection Modes by Optimal Mass Transfer
}

Angelo Iollo, Damiano Lombardi

\section{- To cite this version:}

Angelo Iollo, Damiano Lombardi. Advection Modes by Optimal Mass Transfer. [Research Report] 2012, pp.33. hal-00669693

\section{HAL Id: hal-00669693 \\ https://hal.inria.fr/hal-00669693}

Submitted on 13 Feb 2012

HAL is a multi-disciplinary open access archive for the deposit and dissemination of scientific research documents, whether they are published or not. The documents may come from teaching and research institutions in France or abroad, or from public or private research centers.
L'archive ouverte pluridisciplinaire HAL, est destinée au dépôt et à la diffusion de documents scientifiques de niveau recherche, publiés ou non, émanant des établissements d'enseignement et de recherche français ou étrangers, des laboratoires publics ou privés. 


\title{
Advection Modes by Optimal Mass Transfer
}

\author{
A. Iollo*and D. Lombardi ${ }^{\dagger}$
}

\begin{abstract}
Classical model reduction techniques approximate the solution of a physical model by a limited number of global modes. These modes are usually determined by variants of principal component analysis. Global modes can lead to reduced models that perform well in terms of stability and accuracy. However, when the physics of the model is mainly characterized by advection, the non-local representation of the solution by global modes essentially reduces to a Fourier expansion. In this paper we describe a method to determine a low-order representation of advection. This method is based on the solution of Monge-Kantorovich mass transfer problems. Examples of application to point vortex scattering, Korteweg-de Vries equation, Von Kármán wake and hurricane Dean advection are discussed.
\end{abstract}

\section{Introduction}

Principal Component Analysis (PCA) is the main tool behind many techniques to perform model reduction of systems of partial differential equations (PDEs). The objective of model reduction is to obtain a simpler model that retains the main dynamical features of the full model, typically for optimization and control purposes. The principle of PCA is to find the base of a small dimensional subspace in such a way that the solution of a given PDE is accurately represented in this subspace. This is the main idea behind Proper Orthogonal Decomposition (POD) [7, 10]: for a given database of model solutions, POD

\footnotetext{
*angelo.iollo@math.u-bordeaux1.fr, Univ. Bordeaux, IMB, UMR 5251, F-33400 Talence, France. CNRS, IMB, UMR 5251, F-33400 Talence, France. INRIA, F-33400 Talence, France.

†damiano.lombardi@inria.fr, INRIA, Paris Rocquencourt, B.P.105, 78153 Le Chesnay
} 


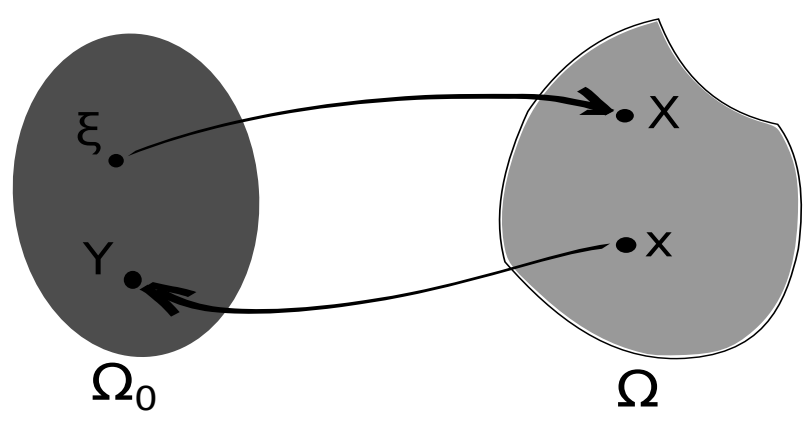

Figure 1: Lagrangian description of transport: the reference configuration is $\Omega_{0}$, points $\xi \in \Omega_{0}$ are transported by the direct mapping in $X(\xi, t)$. Given the actual configuration $\Omega$, a point $x \in \Omega$ is sent back to its counterimage in the reference configuration by backward characteristics, i.e., the inverse mapping $Y(x, t)$.

builds a basis that minimizes the $L^{2}$ average distance between the reduced representation and the solution database. POD approximations are usually satisfactory for those problems where the solution has a global behavior, but tends to perform poorly for systems characterized by concentrated structures that are advected.

Alternatives to POD taking implicitly into account the notion of transport in the definition of a global reduced basis are provided by Koopman modes [6, 9] and Dynamic Mode Decomposition (DMD) [8]. These approaches assume the existence of a linear propagator (relative to some possibly non-linear map) whose spectrum provides a frequency based mode decomposition of the considered evolution. Starting from snapshots of some observables of the physical system, an Arnoldi-type algorithm is employed to estimate the linear propagator.

Our objective here is to introduce the notion of transport modes to represent by a reduced basis the evolution of coherent structures when advection is the leading phenomenon. To plainly present the main ideas let us proceed in two steps.

Transport. In Fig. 1 a conceptual description of transport is shown. Given a point $\xi \in \Omega_{0}$, where $\Omega_{0} \subseteq \mathbb{R}^{d}$ is a reference configuration, transport at time $t$ is described by a mapping $X(\xi, t)$. The point $x=X(\xi, t)$ belongs to the actual physical configuration $\Omega \subseteq \mathbb{R}^{d}$. Let us consider a point $x$ in the actual physical configuration. The inverse mapping, denoted by $Y(x, t)$ (called otherwise backward characteristics), identifies the point in the reference configuration that has been transported by the direct map in $x$ at 
time $t$. The following relations hold:

$$
\begin{aligned}
& x=X(\xi, t), \quad \xi=Y(x, t), \\
& Y=X^{-1}, \quad\left[\nabla_{\xi} X\right]\left[\nabla_{x} Y\right]=I,
\end{aligned}
$$

where $\left[\nabla_{\xi} X\right]$ is the jacobian of the transformation $X(\xi, t)$ and $\left[\nabla_{x} Y\right]$ its inverse, i.e., the jacobian of the inverse mapping. Also, we have:

$$
\begin{aligned}
& \partial_{t} Y+\mathbf{v} \cdot \nabla_{x} Y=0, \quad Y(x, 0)=x \\
& \mathbf{v}(x, t)=\partial_{t} X, \quad X(\xi, 0)=\xi
\end{aligned}
$$

where $\mathbf{v}$ is the velocity field.

Let us consider, as an example, the inviscid Burgers equation:

$$
\partial_{t} \mathbf{v}+\mathbf{v} \cdot \nabla_{x} \mathbf{v}=0
$$

This equation describes a pressureless Euler flow. Since no force is acting on the medium, each component of the velocity field is purely advected. In lagrangian coordinates we have:

$$
\partial_{t}^{2} X(\xi, t)=0 \Longrightarrow X(\xi, t)=\xi+\mathbf{v}(\xi, 0) t .
$$

The solution consists of particles moving on straight lines (no acceleration).

Advection modes. An optimal subspace of given size to represent a solution database is usually sought by minimizing the distance (to be defined) between the solution database and its projection in the subspace. Let $u(x, t)$ represent the solution database, where $x$ and $t$ stand here for discrete time and space. With POD, the distance to be minimized is defined by the norm of an appropriate Hilbert space, and the projected database is represented by

$$
\hat{u}(x, t)=a_{i}(t) \phi_{i}(x), \quad i=1, \ldots, N_{P O D},
$$

where here and in the following we use the summation convention. The function $\phi_{i}$ is called the $i$-th POD mode, and it is computed as a linear combination of the elements of the database, i.e., the snapshots. The solution $u(x, t)$ is approximated by global fields (defined everywhere in the actual physical configuration) which are typically oscillating in time. 
Let now $\tilde{u}$ be another approximation of the database such that:

$$
\tilde{u}(x, t)=\left[u_{0}(Y(x, t))+R(Y(x, t), t)\right] \operatorname{det}\left(\nabla_{x} Y(x, t)\right),
$$

where $u_{0}(\xi)$ is a reference mode, $Y(x, t)$ is a suitable backward mapping and $R(\xi, t)$ is a residual to be defined in the following. This relation states that the database elements can be represented as the sum of a mapped constant reference configuration $u_{0}(\xi)$, and the mapping of a time-dependent residual field $R(\xi, t)$ in the reference configuration.

Three elements have to be defined in order to obtain a reduced representation: a reference mode $u_{0}(\xi)$, a reduced-order expansions for the inverse characteristics $Y(x, t)$ and the residual $R(\xi, t)$.

The following ansätze are introduced:

$$
\begin{aligned}
& R(\xi, t)=\alpha_{k}(t) \phi_{k}(\xi), k=0, \ldots, N_{r} \\
& Y(x, t)=\beta_{j}(t) W_{j}(x), j=0, \ldots, N_{y},
\end{aligned}
$$

where $\phi_{k}$ are POD modes for the residual $R$ and $W_{j}$ are the advection modes to be found.

The rest of this paper explains how to extract the reference mode, the advection modes as well as residual modes starting from a snapshots database $u_{i}=u\left(x, t_{i}\right), i=1, \ldots, N_{s}$.

\section{Advection mode expansion}

In order to determine the advection modes, we define a suitable optimal transport problem. Let us associate a scalar density function $\varrho(u) \geq 0$ to the solution $u(x, t)$, in such a way that:

$$
\int_{\Omega} \varrho(x, t) d x=1, \forall t \in \mathbb{R}^{+}
$$

so that the non-negative density is normalized to 1 for all times. The choice of the density function is for the moment arbitrary. If $u$ is a non-negative scalar and satisfies this normalization, it may be directly used as a density function.

Let $\varrho_{i}, i=1, \ldots, N_{s}$ be the snapshots of the density function. The Wasserstein distance $($ denoted by $\mathcal{W}$ ) relative to a density pair is defined as:

$$
\begin{aligned}
& \mathcal{W}^{2}\left(\varrho_{i}, \varrho_{j}\right)=\inf _{\tilde{X}}\left\{\int_{\Omega} \varrho_{i}(\xi)|\tilde{X}(\xi)-\xi|^{2} d \xi\right\}, \text { subject to } \\
& \varrho_{i}(\xi)=\varrho_{j}(\tilde{X}(\xi)) \operatorname{det}\left(\nabla_{\xi} \tilde{X}\right) .
\end{aligned}
$$


The Wasserstein distance is the distance induced by the optimal mapping $X^{*}$ that minimizes the cost of the $L^{2}$ transport (Monge) problem, among all the change of coordinates $\tilde{X}(\xi)$ locally keeping constant mass between the densities $i$ and $j$. The solution to this problem exists and is unique (see, for a comprehensive review $[11,12,2]$ ).

The squared Wasserstein distance can be computed for all $i, j=1, \ldots, N_{s}: \frac{1}{2} N_{s}\left(N_{s}-1\right)$ Monge problems are solved. Then, the following matrix is defined:

$$
\mathcal{D}_{i j}=\mathcal{W}^{2}\left(\varrho_{i}, \varrho_{j}\right)
$$

that is the matrix of the squared distances between the densities. This matrix is symmetric $\left(\mathcal{W}\left(\varrho_{i}, \varrho_{j}\right)=\mathcal{W}\left(\varrho_{j}, \varrho_{i}\right)\right)$ and all the elements on the diagonal are zero $\left(\mathcal{W}\left(\varrho_{i}, \varrho_{i}\right)=0\right)$.

In an Euclidean vector space, one can uniquely transform a matrix of canonical squared distances relative to a set of points, in a positive semi-definite matrix whose entries are the corresponding scalar products between the position vectors $\chi_{i}$ of those points. In other words

$$
\mathcal{D}_{i j}=\left\|\chi_{i}-\chi_{j}\right\|^{2}=\mathcal{B}_{i i}+\mathcal{B}_{j j}-2 \mathcal{B}_{i j}
$$

where $\mathcal{B}_{i j}=\chi_{i} \cdot \chi_{j}$ are the entries of a matrix $B \in \mathbb{R}^{\mathbb{N}_{s} \times \mathbb{N}_{s}}$. Let $\chi \in E \subseteq \mathbb{R}^{\mathbb{N}_{s}}$ and let $\mathbb{1} \in \mathbb{R}^{N_{s}}$ be a column vector whose components are all 1 . We assume that the vectors representing the points are centered in the origin of the vector space, i.e., that $B \mathbb{1}=0$. Then Eq. (11) can be inverted and we have

$$
B=-\frac{1}{2} J D J
$$

and

$$
J=I-\frac{1}{N_{s}} \mathbb{1}^{T},
$$

where $I$ is the identity matrix. We assumed that $E$ is embedded in an Euclidean space. In this case it can be shown that Eq. 12 leads to a matrix $B$ that is positive semidefinite. For a Wasserstein distance matrix this is not necessary the case. However, one can look for an euclidean set of vectors that gives a squared distance matrix which is an approximation of the Wasserstein square distance matrix.

In order to do this, we adopt the same technique presented in [4]. Matrix $B$ is real and symmetric and hence it can be decomposed as:

$$
B=U S U^{T}
$$


where $U$ is a unitary matrix and $S$ is the diagonal matrix whose entries are the eigenvalues of $B$. Let us take the positive part of $B$

$$
B^{+}=U S^{+} U^{T} \text { where } S^{+}=\frac{S+|S|}{2}
$$

so that $D \approx B^{+},\left\|D-B^{+}\right\|=\left\|\frac{S-|S|}{2}\right\|$ and $B^{+}$is of course positive semidefinite. It is now possible to determine matrix $X$ whose columns are the position vectors $\chi$ as $X=U \sqrt{S^{+}}$. A normalization technique, which is useful when a large number of snapshots is taken is discussed in Appendix.

As stated, the embedding is performed with respect to the origin of the euclidean space, which corresponds to a density distribution whose properties will be investigated in the following sections. The $i-t h$ component of the $k-t h$ eigenvector of $B^{+}$represents the weight of the $i-t h$ mapping (that transports the origin in the $i-t h$ snapshot) to build the optimal transport corresponding to the $k-t h$ eigenvalue. In the following we define the $k-t h$ transport mode as the $k-t h$ optimal transport, once a barycentric density is given. Conversely, when a base of mappings is taken in the space $\left\{v_{1}, \ldots, v_{k}\right\}$, the mapping that transports the barycenter in the $i-t h$ snapshot is derived by summing the base mappings multiplied by the coordinates of the point representing the $i-t h$ snapshot.

In POD the snapshots reduced space is generated by the snapshots themselves, while for transport modes the representation of the snapshots is provided by a set of optimal transports that map a barycentric density into the snapshots. This barycentric density may be used as $u_{0}(\xi)$. The mappings obtained in this way are the basis of transport mappings. Let us remark that two series of optimal mappings are obtained: the direct mappings and their corresponding inverses.

\subsection{Residual representation}

In this section the representation of the function residual function $R$ is discussed. The transport modes are defined according to the technique presented in the section above.

The entire set of transport modes account for an exact representation of the densities. A subset of $N_{T}$ transport modes is chosen, in order to provide a reduced representation 
of the direct and inverse mapping, respectively $X(\xi, t)$ and $Y(x, t)$ :

$$
\begin{aligned}
& X\left(\xi, t_{i}\right)=\alpha_{j}\left(t_{i}\right) Z_{j}(\xi), \\
& Y\left(x, t_{i}\right)=\beta_{j}\left(t_{i}\right) W_{j}(x), j=0, \ldots, N_{T} \leq N_{s},
\end{aligned}
$$

where the coefficient $\alpha_{j}\left(t_{i}\right)$ is known from the components of the $j$-th eigenvector of the embedding matrix $B$ and the coefficient $\beta_{j}$ may be derived by considering the definition of $Y$ as the inverse mapping.

Then we map each snapshot to the reference domain and compute the residual with respect to the initial condition

$$
R_{i}(\xi)=R_{i}\left(X\left(\xi, t_{i}\right)\right)=u\left(X\left(\xi, t_{i}\right), t_{i}\right) \operatorname{det}\left(\nabla_{\xi} X\left(\xi, t_{i}\right)\right)-u_{0}(\xi),
$$

where $u\left(x, t_{i}\right)=u\left(X\left(\xi, t_{i}\right), t_{i}\right)$ are the solution snapshots. Then the classical POD technique is applied:

$$
A_{i j}=\int_{\Omega_{0}} R_{i}\left(X\left(\xi, t_{i}\right)\right) R_{j}\left(X\left(\xi, t_{j}\right)\right) d \xi
$$

and the POD residual modes are defined through the eigenvalues and eigenvectors of the autocorrelation matrix:

$$
\phi_{k}(\xi)=\frac{\sum_{h} b_{h}^{(k)} R_{h}(\xi)}{\lambda_{k}^{1 / 2}},
$$

where $b_{h}^{(k)}$ is the $h$-th component of the $k$-th eigenvector of $A$ and $\lambda_{k}$ its corresponding eigenvector.

We finally obtained a representation in a reference configuration of a time dependent field, decomposed in a mean field (the barycentric density or equivalently the initial condition) and a POD expansion. This time dependent field is mapped to an actual configuration at time $t_{i}$ by the mapping $Y\left(x, t_{i}\right)$, determined through a decomposition based on the Wasserstein distance. In order to actually compute the Wasserstein distance matrix in this work we refer to the technique presented in [3], where the optimal mass transfer problem is solved by a lagrangian method based on the fact that characteristics reduces to straight lines.

\section{$3 \quad$ Numerical experiments}

In this section we present four illustrations of increasing complexity ranging from an exact idealized case to experimental data. 


\subsection{Ideal vortex scattering}

We consider two couples of counter-rotating ideal point vortices in the plane. The flow is incompressible and the vorticity is represented by four Dirac masses located at the vortex centers, so that the flow is irrotational almost everywhere. Under these hypotheses the flow is potential and the trajectories of the point vortices are obtained by the solution of an hamiltonian dynamical system. The detailed derivation of the governing equations is found in $[5]$.

The flow domain is $\mathbb{R}^{2}$ and the coordinates of the vortex cores are:

$$
\begin{aligned}
& x_{a}=r_{1} \cos \left(\theta_{1}\right) \quad y_{a}=r_{1} \sin \left(\theta_{1}\right), \\
& x_{b}=r_{2} \cos \left(\theta_{2}\right) \quad y_{b}=r_{2} \sin \left(\theta_{2}\right), \\
& x_{c}=r_{1} \cos \left(\theta_{1}+\pi\right) \quad y_{c}=r_{1} \sin \left(\theta_{1}+\pi\right), \\
& x_{d}=r_{2} \cos \left(\theta_{2}+\pi\right) \quad y_{d}=r_{2} \sin \left(\theta_{2}+\pi\right),
\end{aligned}
$$

where $r_{1}, r_{2}, \theta_{1}$, and $\theta_{2}$ are the only variables necessary to describe the interaction. They are initialized as follows:

$$
\begin{aligned}
& r_{1}(0)=\left(l^{2}+(1+\beta) f^{2}\right)^{\frac{1}{2}}, \\
& r_{2}(0)=\left(l^{2}+(1-\beta) f^{2}\right)^{\frac{1}{2}}, \\
& \theta_{1}(0)=\arctan \left[\frac{(1+\beta) f}{l}\right], \\
& \theta_{2}(0)=\arctan \left[\frac{(1-\beta) f}{l}\right],
\end{aligned}
$$

where $l, \beta$ and $f$ are three parameters that determine the distance of the dipoles, the relative distance of the counter rotating vortices and the offset of the dipole axis. The initial geometry of the system determines the nature of the scattering occurring between the dipoles. The ODEs describing the evolution are:

$$
\begin{aligned}
& \dot{r}_{1}=-\frac{2 \sin \left(2\left(\theta_{1}-\theta_{2}\right)\right) r_{1} r_{2}^{2}}{\pi\left(r_{1}^{4}-2 \cos \left(2\left(\theta_{1}-\theta_{2}\right)\right) r_{1}^{2} r_{2}^{2}+r_{2}^{4}\right)}, \\
& \dot{r}_{2}=-\frac{2 \sin \left(2\left(\theta_{1}-\theta_{2}\right)\right) r_{2} r_{1}^{2}}{\pi\left(r_{1}^{4}-2 \cos \left(2\left(\theta_{1}-\theta_{2}\right)\right) r_{1}^{2} r_{2}^{2}+r_{2}^{4}\right)}, \\
& \dot{\theta}_{1}=\frac{3 r_{1}^{4}-2 \cos \left(2\left(\theta_{1}-\theta_{2}\right)\right) r_{1}^{2} r_{2}^{2}-r_{2}^{4}}{2 \pi r_{1}^{2}\left(r_{1}^{4}-2 \cos \left(2\left(\theta_{1}-\theta_{2}\right)\right) r_{1}^{2} r_{2}^{2}+r_{2}^{4}\right)}, \\
& \dot{\theta}_{2}=\frac{r_{1}^{4}+2 \cos \left(2\left(\theta_{1}-\theta_{2}\right)\right) r_{1}^{2} r_{2}^{2}-3 r_{2}^{4}}{2 \pi r_{2}^{2}\left(r_{1}^{4}-2 \cos \left(2\left(\theta_{1}-\theta_{2}\right)\right) r_{1}^{2} r_{2}^{2}+r_{2}^{4}\right)} .
\end{aligned}
$$


The equations of motion are integrated via an adaptive-step fourth-order Runge-Kutta scheme in the time interval [0,2.5]. In Fig. 2, three different situations are represented: a) a scattering where the vortices keep their partner (the parameters used are: $l=1.5$, $\beta=0.5, f=0.25)$; b) a case where the vortices exchange their partner and escape with the counter rotating vortex belonging to the other dipole $(l=1.0, \beta=0.75, f=0.15)$; ) a weak interaction in which the dipoles simply move on (almost) straight lines $(l=2.0$, $\beta=0.15, f=0.30)$.

We consider the $L^{2}$ norm of vorticity (enstrophy) as the transported density $\varrho$. In this motion enstrophy is constant, so that we analyse the dynamics of four unitary Dirac masses. The Wasserstein distance between the time snapshots was computed by means of an exact combinatorial algorithm. For all the following cases 50 time frames were taken between initial and final time.

The embedding technique presented in the previous section was adopted. The spectrum of matrix $B$ includes a few negative eigenvalues due to the fact that the distance is not euclidean. They are small in modulus so that $B \approx B^{+}$. In Fig. 3 the eigenvalues of the embedding matrix are represented for the first case described (a). Only two eigenvalues are relevant in the approximation of the phenomenon. The corresponding eigenvectors are represented in a phase-plane plot. The circles represent the components of the eigenvectors and can be associated to the time frames. Two directions that represent the optimal transports occurring before and after the interaction can be observed. The points which are not aligned represents the snapshots of the enstrophy configurations occurring during the interaction.

For case (b) (see Fig. 4) the spectrum of the embedding matrix is similar to that obtained for the first case: two eigenvalues emerge. The phase plot of the first two eigenvectors shows that the vortex interaction is quite different, but again two main transport direction corresponding to the dynamics before and after the interaction are present.

The third case (see Fig. 5) is different from the others. The interaction is weak and the resulting motion is practically an optimal transport. This third case may be considered as a perturbation of a single optimal transport. Indeed, in Fig. 5.a) the plot of the eigenvalues confirms that only one eigenvalue is important. The plot of the corresponding eigenvector in Fig. 5.b) show that most of the snapshots are aligned, that is, they may be obtained by non-linear interpolation (i.e., by transport) of the barycentric density via a unique optimal 

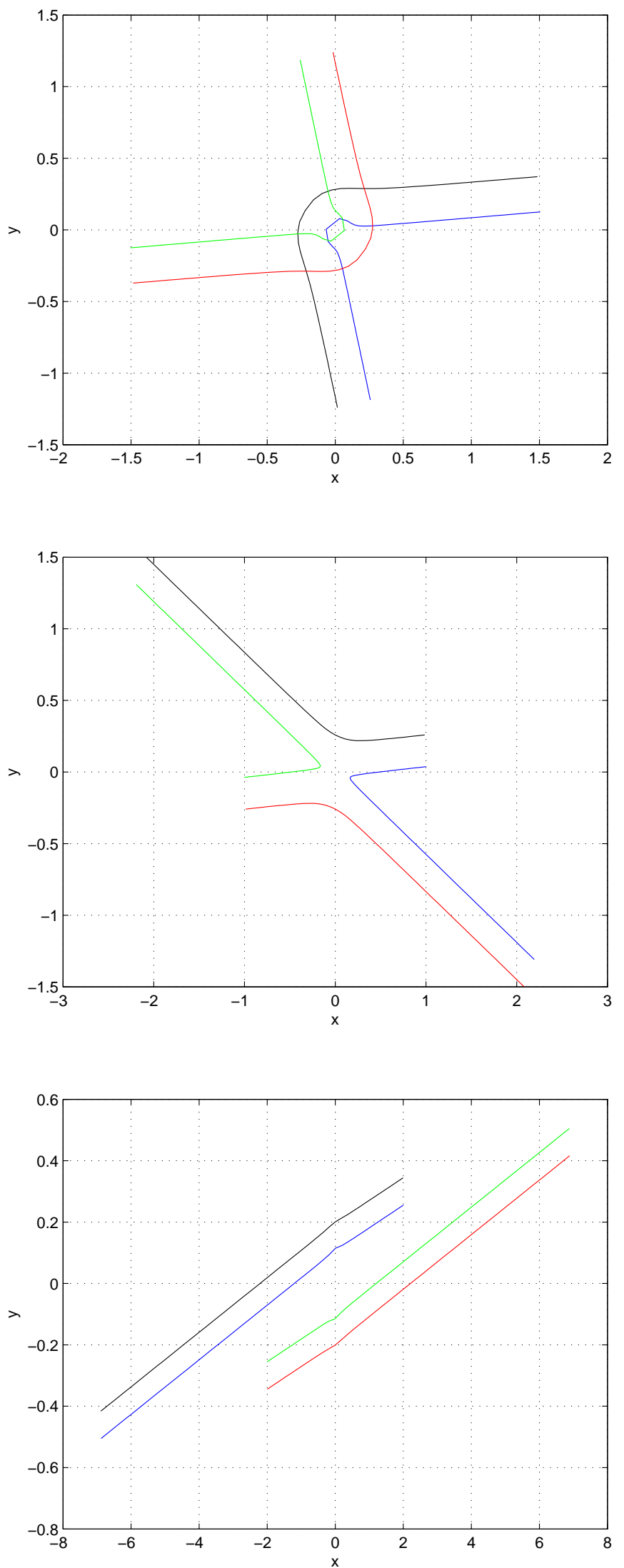

(a) top (b) middle (c) bottom

Figure 2: Three different scattering trajectories of vortex cores for: a) $l=1.5, \beta=0.5$, $f=0.25$ b) $l=1.0, \beta=0.75, f=0.15$ c) $l=2.0, \beta=0.15, f=0.30$. 


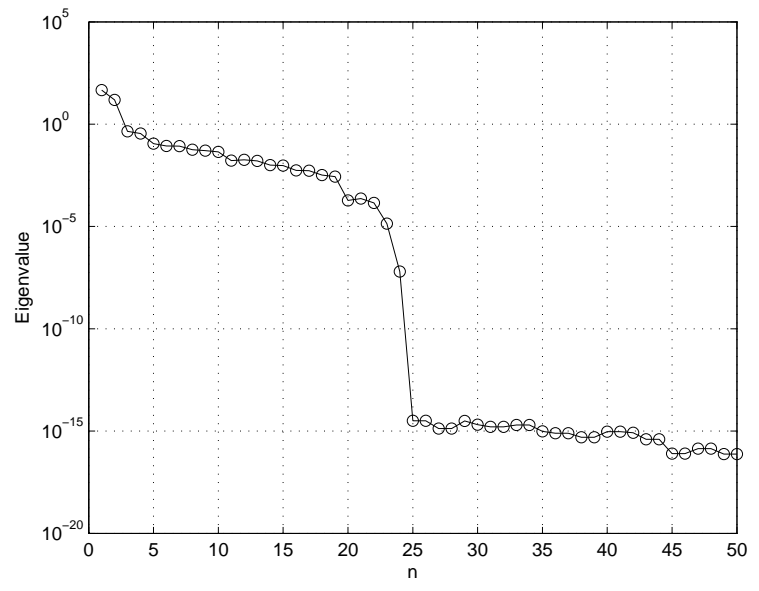

(a)

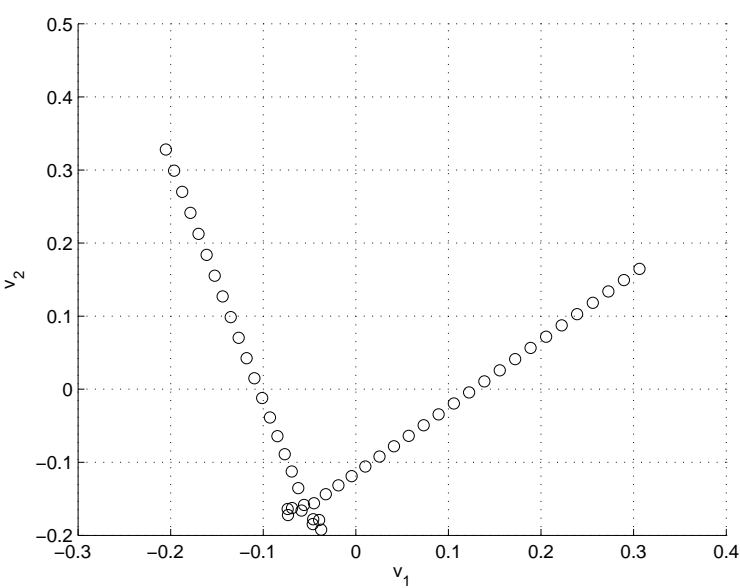

(b)

Figure 3: First case: a) the eigenvalues of the embedding matrix in logarithmic scale; b) the first two eigenvectors are represented in a phase-plane plot.

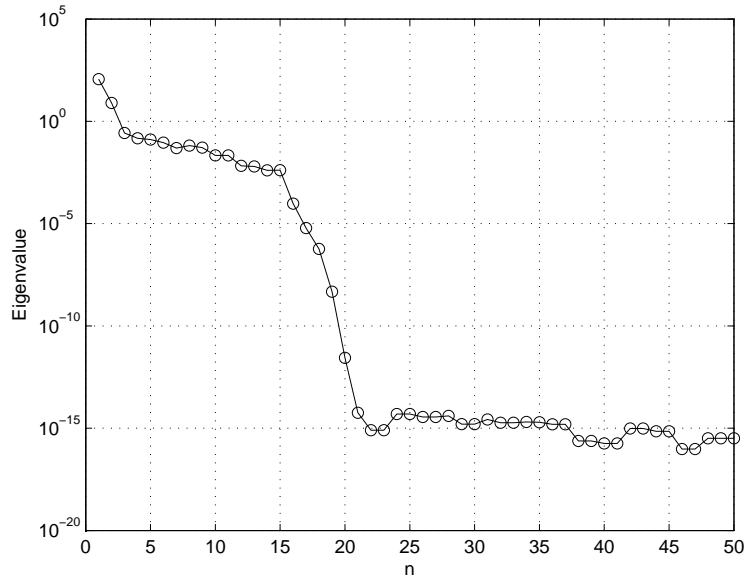

(a)

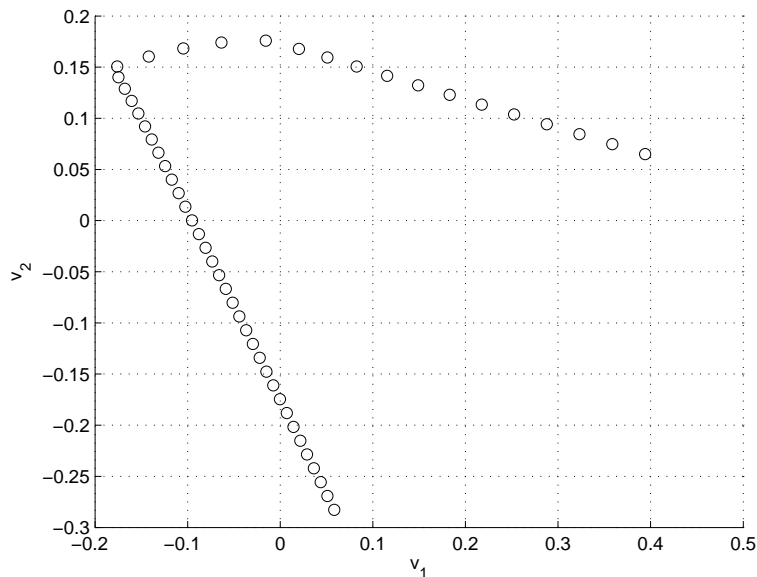

(b)

Figure 4: Second case: a) the eigenvalues of the embedding matrix in logarithmic scale; in b) the first two eigenvectors represented in a phase-plane plot. 


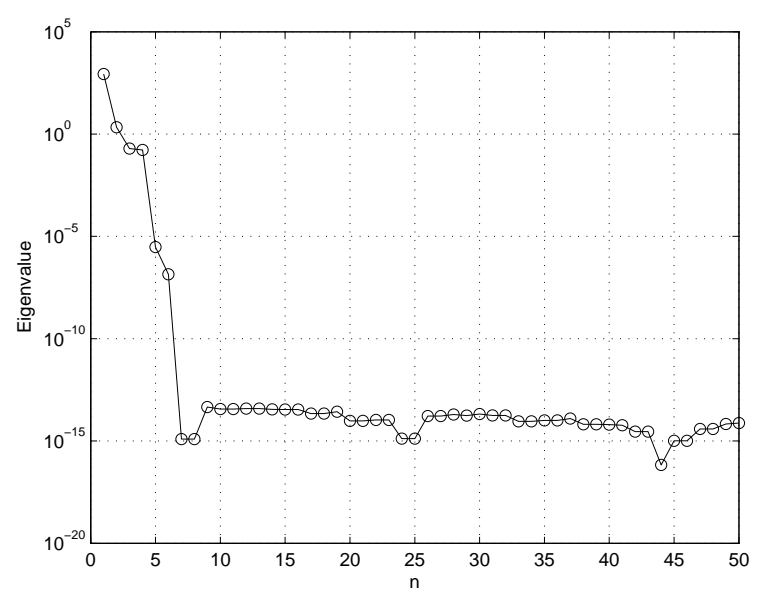

(a)

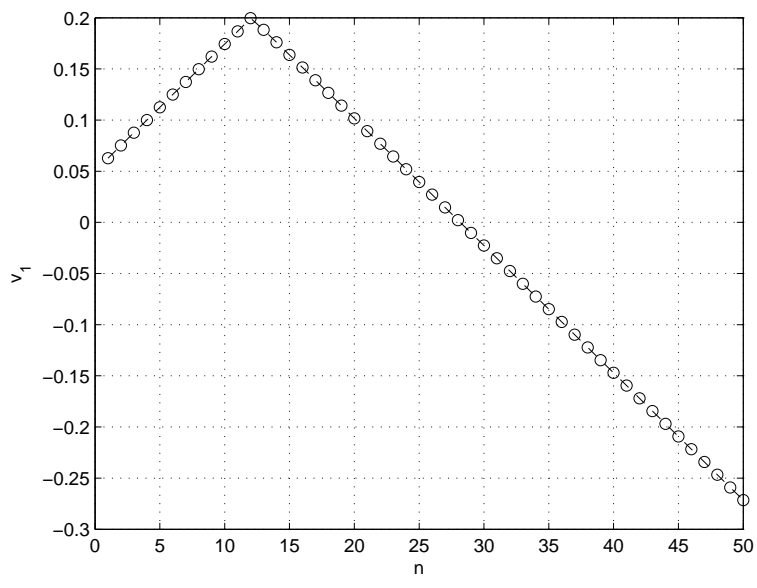

(b)

Figure 5: Third case: a) the eigenvalues of the embedding matrix in logarithmic scale; in b) the first eigenvector.

transport. In this simple example the comparison with standard POD is straight forward. For the second and the third cases (Fig. 2 b) and c)) the autocorrelation matrix (i.e., the matrix of scalar products of the snapshots) is the identity matrix. Hence, no reduction can be provided by using POD. For the first case (Fig. 2.a)), the trajectories intersect, so that the autocorrelation matrix may not be diagonal for some specific samplings. However, only few extradiagonal elements appear, so that, even in this case, no signal reduction is possible.

\subsection{One-dimensional Korteweg-de-Vries equation}

The one-dimensional Korteveg-de-Vries equation is an interesting test case because transport and dispersion can be modulated. The model equation reads:

$$
\partial_{t} u+u \partial_{x} u+\gamma \partial_{x x x} u=0
$$

where $u(x, t)$ is the solution at time $t \in[0,2.5]$ and position $x \in[0,2 \pi], \gamma=1 e-3$ is the dispersion parameter. Periodic boundary conditions are imposed. The initial condition is $u(x, 0)=u_{0}(x)$ and for the present case is taken as:

$$
u_{0}(x)=0.1+\exp \left\{-2\left(x-\frac{\pi}{2}\right)^{2}\right\} .
$$




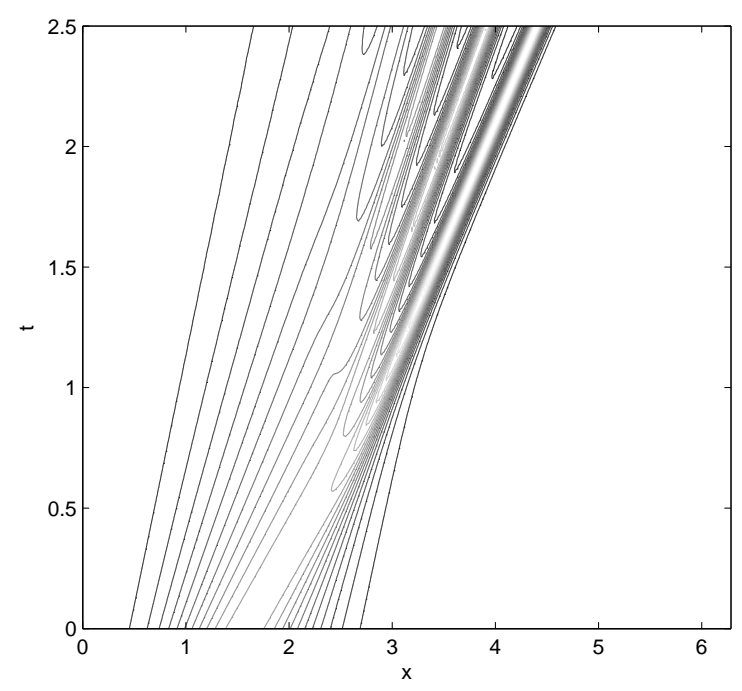

(a)

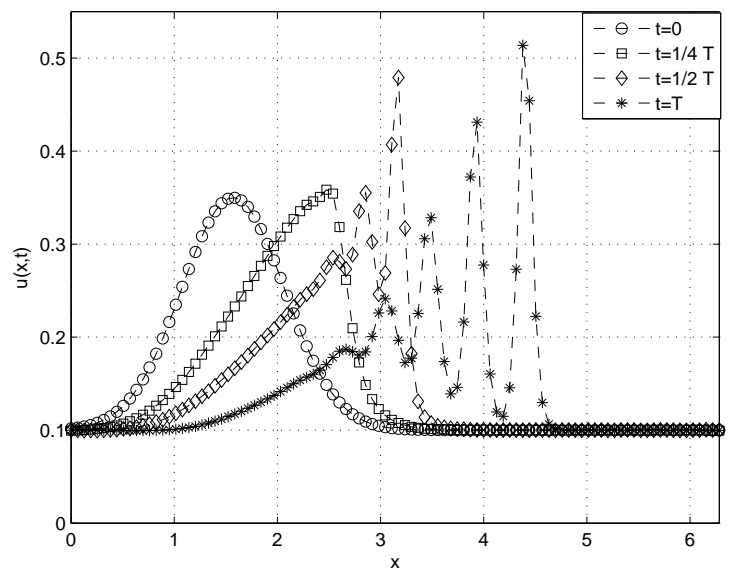

(b)

Figure 6: Solution of the KdV model: a) contour of $\mathrm{x}$-t diagram, 20 values between minimum and maximum b) solution at different times, where $\mathrm{T}=2.5$.

This equation was numerically integrated using a spectral discretization in space (256 Fourier modes), and a second order Cranck-Nicholson method with $10^{3}$ steps in time.

The preserved measure is in this case the energy of the signal. Let $e=u^{2}$, then it can be proved that $\forall t$,

$$
E(t)=\int_{0}^{2 \pi} e(x, t) d x=E(0)=\int_{0}^{2 \pi} u_{0}(x)^{2} d x .
$$

The solutions for this model, shown in Fig. 6, show transport and dispersion. In particular, as $\gamma$ is small, in the first part of the evolution the system evolves as an inviscid Burgers model (Fig. 6.b)). When a shock tends to form, the third derivative norm becomes large and dispersion makes the solution brake in several waves, with different characteristic velocities. For this case, a comparison between POD and the proposed technique is carried out. We take set of 24 snapshots of the solution energy, normalized by its integral (which is constant) between the time $t=0$ and the time $t=T=2.5$.

Let us compute the advection modes defined by means of Wasserstein distance. We computed the optimal transport between all the possible couples of snapshots and hence we formed the embedding matrix as described in the previous sections. The eigenvalues 


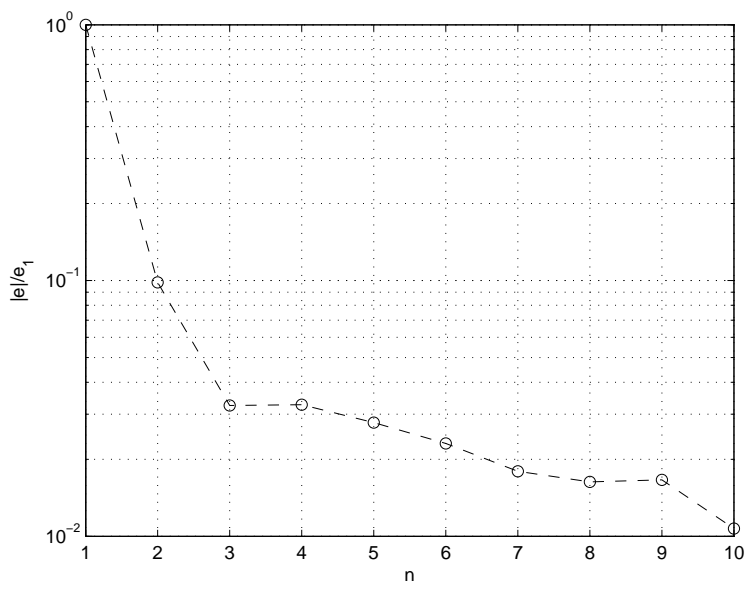

(a)

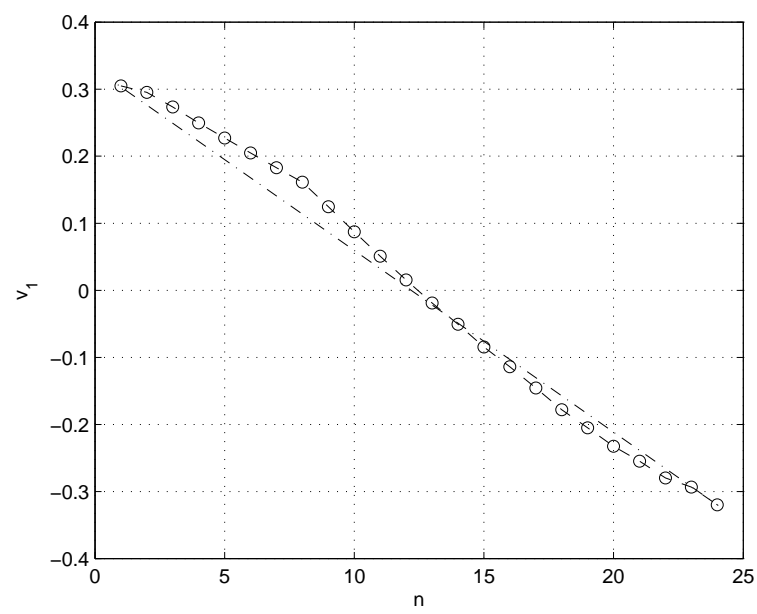

(b)

Figure 7: Embedding matrix analysis: a) first 10 normalized eigenvalues in modulus; b) components of the first eigenvector.

and eigenvectors of this matrix are shown in Fig. 7 a). The absolute value of the first ten eigenvalues is plotted, normalized with the value of the first one: one dominant eigenvalue appears. In Fig. 7.b) the corresponding eigenvector is plotted for each snapshot. The black circles that represent the snapshots are almost aligned, i.e., there exist one optimal transport that approximately interpolate them.

This advection mode may be defined using the straight line connecting the first snapshot with the last one (see Fig. 7.b)), as it is a good interpolant of all the snapshots. This is by definition the optimal mapping that transports the first snapshot, $e(x, 0)$, into the last one $e(x, T)$. Let us denote by $X_{1}(\xi, t)$ this mapping.

The second step of the procedure consists in computing the residuals of this representation. The snapshots $e\left(x, t_{i}\right)$ are mapped back to the initial configuration defined by $X_{1}(\xi, t)$. The differences between the solution snapshots pushed back and $e(x, 0)=e_{0}(\xi)$ is computed by

$$
R_{i}=R\left(\xi, t_{i}\right)=e\left(X_{1}\left(\xi, t_{i}\right), t_{i}\right) \partial_{\xi} X_{1}-e_{0}(\xi),
$$

where $e\left(X_{1}\left(\xi, t_{i}\right), t_{i}\right) \partial_{\xi} X_{1}$ are the snapshots $e_{i}\left(x, t_{i}\right)$ pushed back by the transport mode. These residuals are then decomposed in the reference configuration by means of a standard POD analysis. Let us compare the results of this analysis with that of the POD applied 


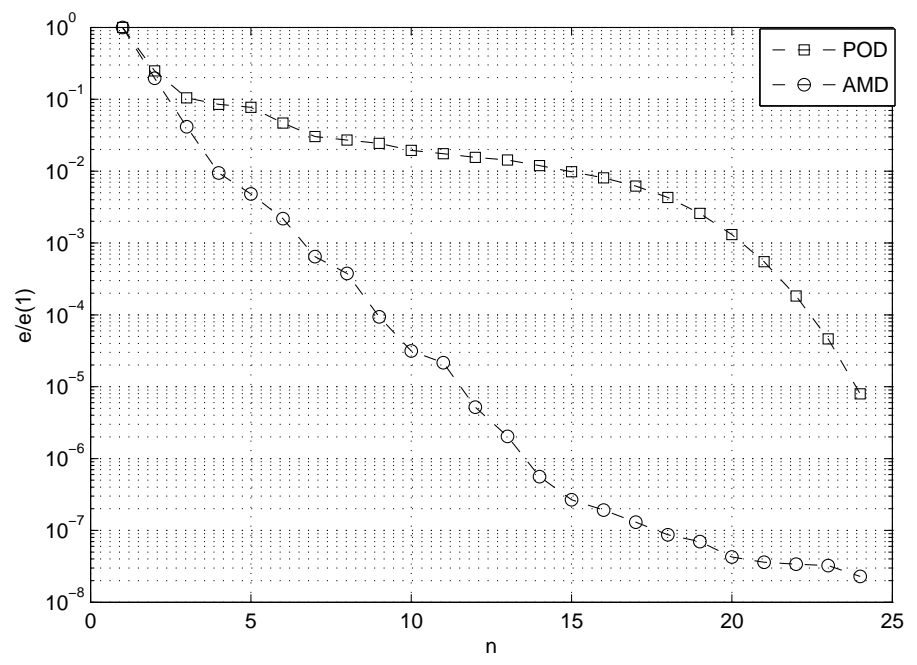

Figure 8: Comparison between standard POD and the POD of the residuals pushed back. Normalized eigenvalues of the autocorrelation matrix

to the original set of snapshots. In Fig. 8 the normalized spectrum of the autocorrelation matrix is shown for the standard POD applied to the snapshots of the energy, and for the POD applied to the residuals pushed back. The eigenvalues cascade for the latter is steeper, i.e., a smaller number of modes is necessary for given representation error.

In Fig. 9.a) the transport mode $U_{1}$ is represented as function of $X_{1}(\xi, 0)=\xi$. The lagrangian coordinate used to pushback the residuals is: $X_{1}(\xi, t)=\xi+t U_{1}(\xi)$. In Fig. 9.b) and c) the first and second residual mode is represented as function of $\xi$. Let us remark that these modes have significant variations in the portion of the domain where the initial energy distribution varies. Let us compare these modes with the classical POD modes for the energy distribution, represented in Fig. 10. The POD modes are quite different from the residual modes: they are global and their support approximately extends to the whole domain. We compare now the reconstruction of the snapshots using the advection mode decompoition (AMD) and POD. The same number of modes is used for both the techniques. Hence, for the POD reconstruction we will use one additional mode compared to the number of residual modes. This allows a fair comparison because for the advection mode decomposition we also have (in this case) one transport.

Three different reconstructions are considered using 4,6 and 10 POD modes respectively. In Fig. 11 the $L^{2}$ normalized errors are plotted as function of the snapshot number 

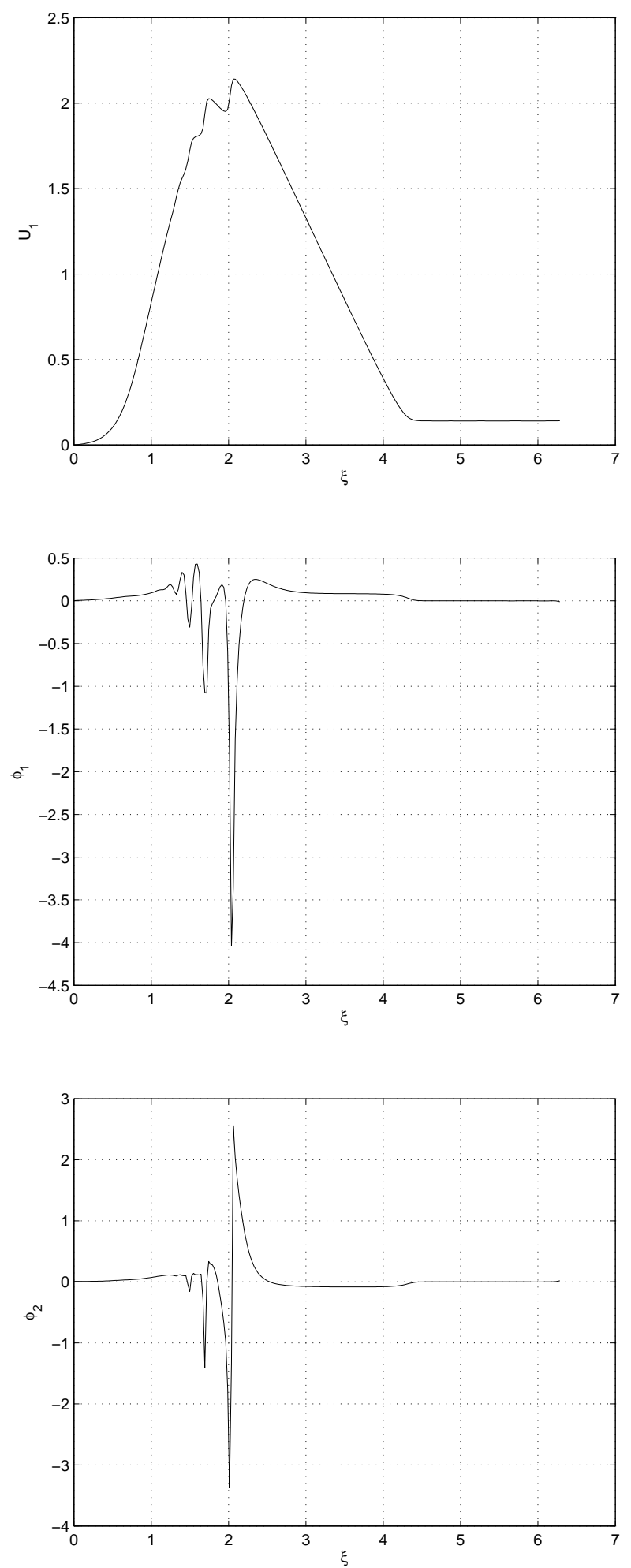

(a) top (b) middle (c) bottom

Figure 9: Advection modes expansion for KdV model: a) Transport mode b) First mode of the residuals c) Second mode of the residuals. 

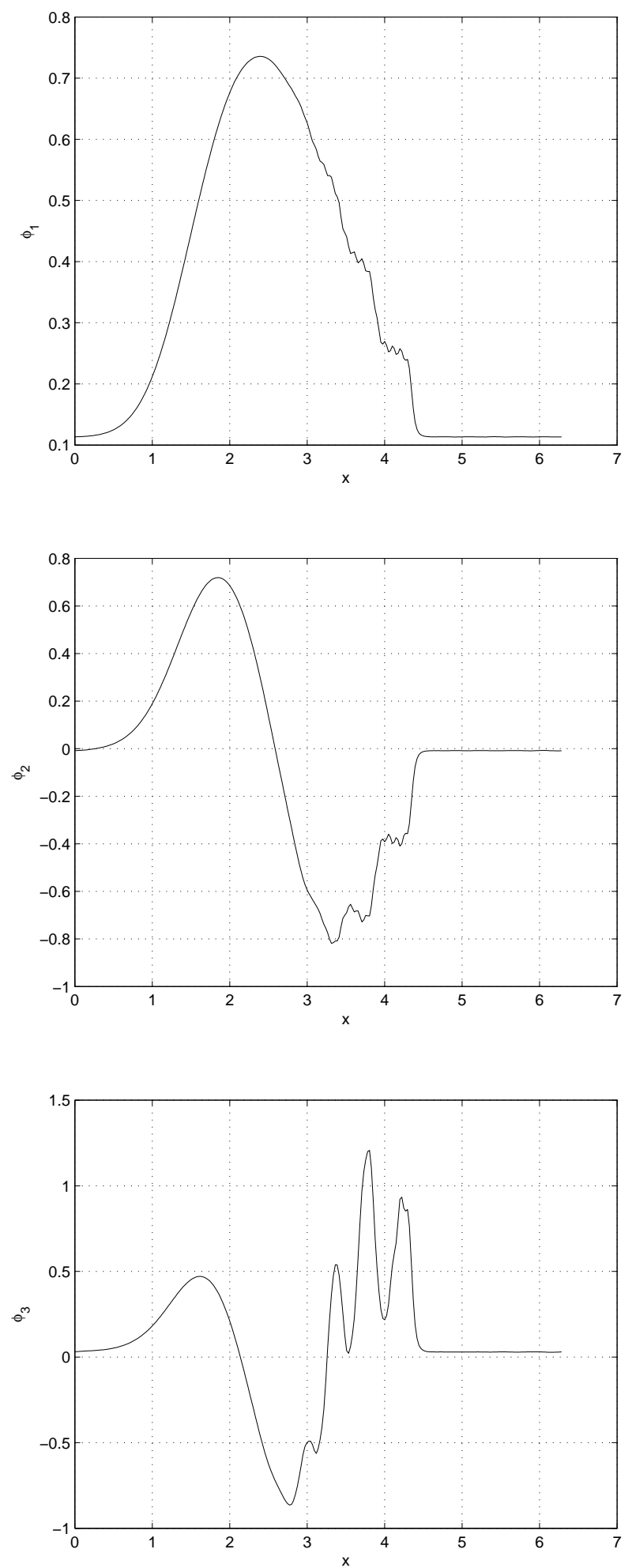

(a) top (b) middle (c) bottom

Figure 10: POD modes for the normalized kinetic energy: a) First mode b) Second mode; c) Third mode. 

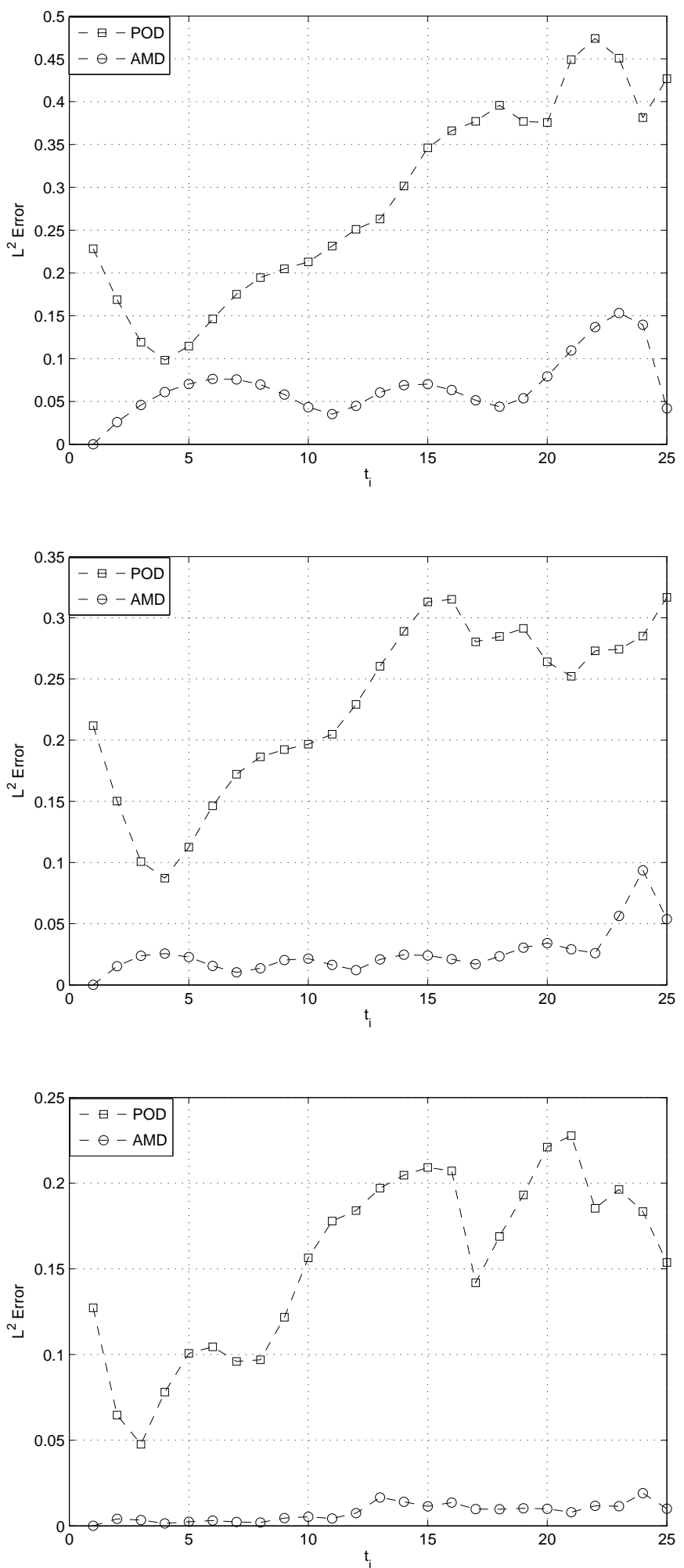

(a) top (b) middle (c) bottom

Figure 11: $L^{2}$ errors as a function of the snapshot number: a) Four modes b) Six modes c) Ten modes 
for POD and advection mode decomposition. In all three cases the advection mode decomposition shows an error that is significantly smaller with respect to that of POD. Also, it tends to diminish faster as the number of modes is increased. This could be anticipated from the spectrum decay. The reconstructions in the physical space for both methods are compared to the snapshot for which the representation given by advection mode decomposition is worst, that is $n=23$.

When few modes are used, like in Fig. 12.a), POD is not able to reproduce the peaks that characterize the solution. In the cases shown in Fig. 12.b-c) POD provides a nonphysical solution due to its essentially oscillatory nature: the reconstructed kinetic energy is negative in a few regions of the domain. The advection mode decomposition in Fig. 12.b) is such that all the key features of the solution are well reproduced. In Fig. 12.c) the agreement is even improved.

\subsection{Two-dimensional vortex shedding}

In this section the vortex shedding past a confined circular cylinder is analyzed. We consider here the kinetic energy of the flow as the transported density. For each snapshot this quantity has been normalized in order to fulfill the constant mass constraint. In this case the normalization does not considerably affect the energy distribution since for this flow its integral is practically constant. Half a period of vortex shedding is considered. The Reynolds number is $R e=200$. The simulations are performed by a standard state of the art simulation code.

We took 10 snapshots of the normalized kinetic energy of the flow and computed the Wasserstein squared distance matrix. To do so, the space resolution adopted was $200 \times 100$, resulting in $2 \cdot 10^{4}$ collocation points. We used a multilevel scheme with 4 grids and linear interpolation kernels to compute the Wassestein distance. The core of this method is similar to what presented in [3] In Fig. 13.a) the first 9 normalized eigenvalues (in absolute value) of the embedding matrix associated to the problem are shown. The cascade is less steep with respect to the one observed for the vortex scattering and the Korteweg-de-Vries equation. However, even in this case a small number of modes may be retained since there are two dominant eigenvalues, see Fig. 13.a). In Fig. 13.b) the phase plot of the first two eigenvectors is shown, revealing a remarkable structure: the points are located on a circle. This means that, given two orthogonal mappings $\phi_{1}, \phi_{2}$ the flow is 

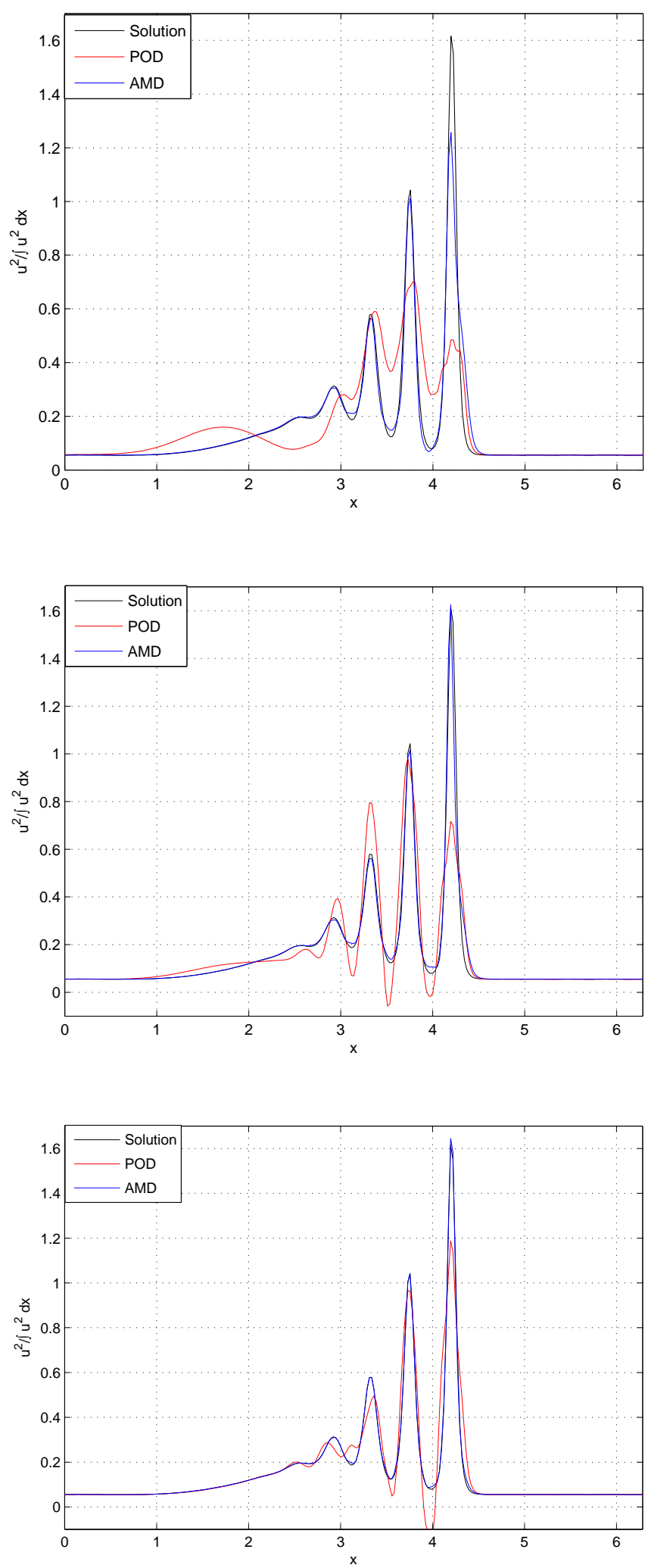

(a) top (b) middle (c) bottom

Figure 12: Reconstruction of the solution. Black line is the solution, blue line is the advection mode decomposition, red line is POD representation when: a) 4 modes, b) 6 modes, c) 10 modes are used 


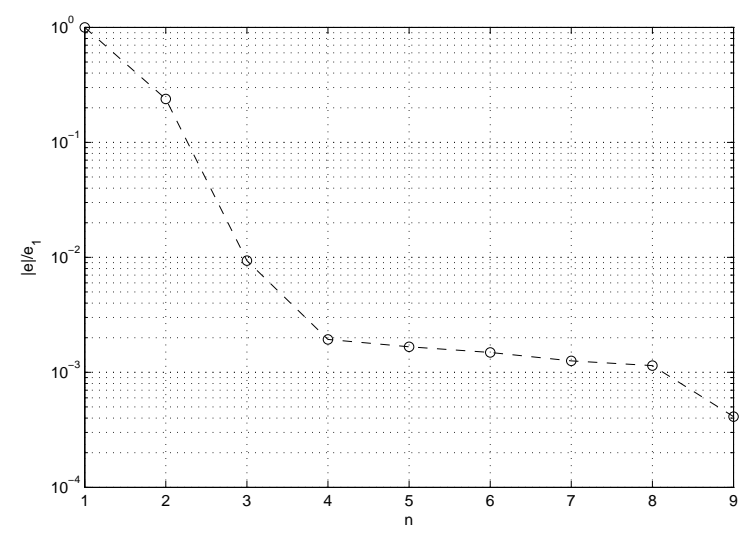

(a)

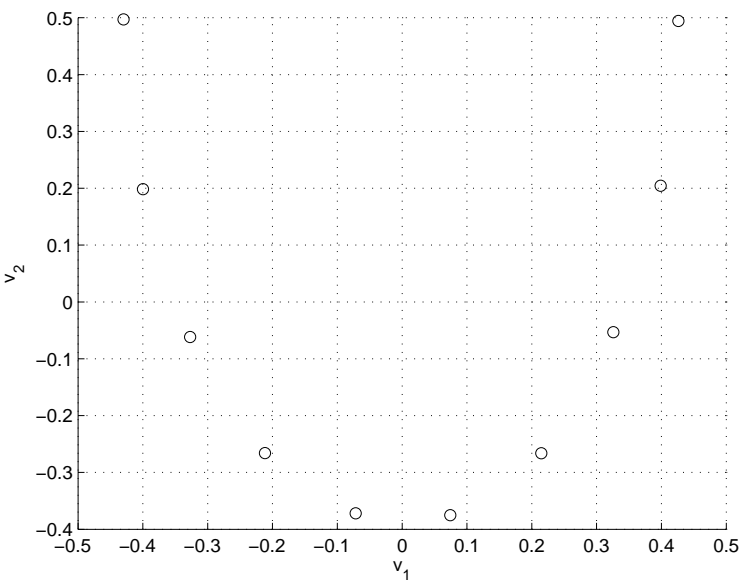

(b)

Figure 13: Analysis of the spectrum of the embedding matrix for the kinetic energy of the flow around a circular cylinder: a) eigenvalues in logarithmic scale b) phase plot of the first two eigenvectors

well approximated by the transport of a barycentric density (localized at the center of the circle) by the following mapping: $\Phi(\xi, t)=\cos (2 \pi t) \phi_{1}(\xi)+\sin (2 \pi t) \phi_{2}(\xi)$, where $t \in[0,1]$ is the time corresponding to one period.

This analysis suggests that three snapshots are sufficient to compute the barycentric density and two orthogonal base mappings. Three snapshots are taken at $t=0, t=0.25$, $t=0.5$, equally distributed on half a period. In the following $\varrho_{0}$ is the kinetic energy distribution at the very beginning, $\varrho_{1}$ the kinetic energy at a quarter of period and $\varrho_{2}$ that of at half a period. The optimal transport is computed by means of the multilevel algorithm, then, the obtained mapping is used to transport $\varrho_{0}$ into the barycentric one, according to:

$$
X_{G}=\xi+\frac{1}{2} \nabla_{\xi} \phi_{2}
$$

where $\phi_{2}$ is the optimal transport from $\varrho_{0}$ to $\varrho_{2}$ and the factor $1 / 2$ means that the collocation points are moved by half the displacement that allows to map $\varrho_{0}$ into $\varrho_{2}$. Hence:

$$
\varrho_{0}(\xi)=\varrho_{G}\left(X_{G}\right) \operatorname{det}\left(\nabla_{\xi} X_{G}\right) .
$$

In Fig. 14 the barycentric density is shown, computed from the nonlinear interpolation 


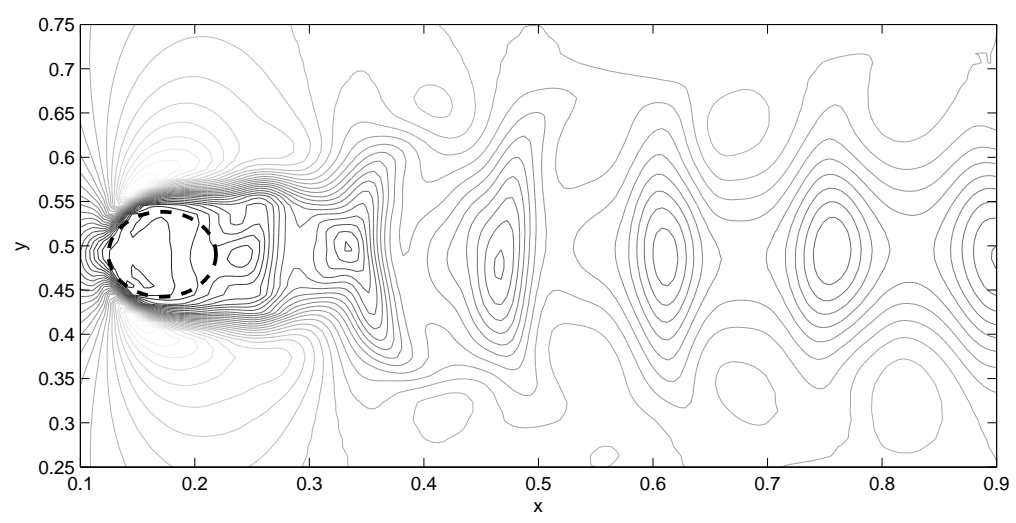

Figure 14: Barycentric density for the kinetic energy of the flow around a circular cylinder: isocontours, 30 lines between the maximum and the minimum

between the kinetic energy distributions $\varrho_{0}$ and $\varrho_{2}$. It is not perfectly symmetrical with respect to the $x$ axis, and this is due to the fact that the considered snapshots have a slight asymmetry too. Let us remark that the barycentric density is not a configuration happening in the physical evolution of the system and it is not the snapshot average. However, the average position of the structures and the characteristic distance of the vortex may be observed. In Fig. 15 the base mappings are shown. The first mapping (Fig. 15.a) has already been computed to find the barycentric density. Once obtained, the mapping $\phi_{1}$ between the barycentric density and $\varrho_{1}$ (i.e. the density located at a quarter of period) is computed thus providing the mapping represented in Fig. 15.b). The displacements fields (computed by taking the gradient of the potentials) are two sequences of alternated couples of sources and sinks that generate the periodicity of the structures.

Given the base maps, the snapshots of the flow are reconstructed by transporting the barycentric density with the suitable displacement field, obtained by summing the mappings multiplied by $\left(\cos \left(2 \pi t_{i}\right), \sin \left(2 \pi t_{i}\right)\right)$, where $t_{i}$ is the position in the period of the $i-t h$ snapshot.

Two cases are shown, corresponding to the best and to the worst approximation. In Fig. 16.a) the representation is shown for $t=0$. All the structures are represented correctly in terms of position and intensity. In Fig. 16.b) the worst approximation is 


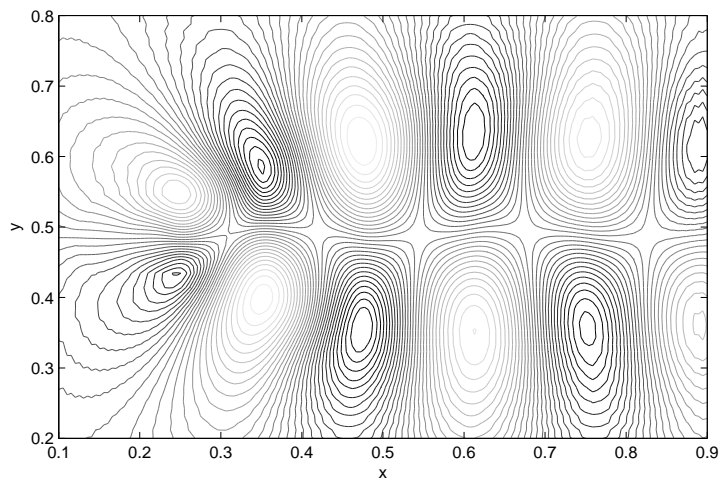

(a)

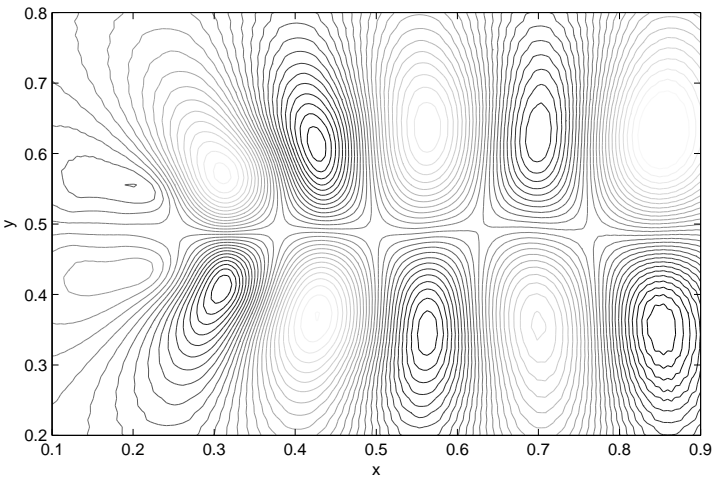

(b)

Figure 15: Isocontours of the base mappings: 30 lines between the maximum $(1.25 e-3)$ and the minimum $-1.25 e-3$.

shown corresponding to the kinetic energy at $t=1 / 8$, which is the farthest from the snapshots used to build the reduced space. Some errors appear concerning the position and the intensity of the structures. However, these errors are localized in the wake. The structures near the body are well captured. This test case shows that a sufficiently good approximation of the flow patterns can be inferred by using little of information about the flow. The representation adopted is the minimal one, consisting in two advection modes computed using three snapshots.

As previously done for the Korteweg-de-Vries equation, the residual pushed back to the reference configuration (in this case the barycentric density) has been computed and the results are compared to classical POD applied to the snapshots sequence. In Fig. 17.a) the spectrum of the autocorrelation matrix of the residuals pushed back is compared to that of the autocorrelation matrix of the POD technique applied to the snapshots. After the third eigenvalue the two curves become superposed. This is explained by the fact that two transport modes have been used and they have exactly the same effect of the first two POD modes. Adding modes for the residual to the advection mode representation or using standard POD is practically equivalent. Since we took snapshots of a periodic solution, a representation of kinetic energy given as global fields that periodically oscillate is equivalent to that of a periodic transport that maps a fixed distribution. No advantage appears in this case in the use of advection mode decomposition to reconstruct the data. However, only 3 flow snapshots were used to recover the transport mappings and hence 


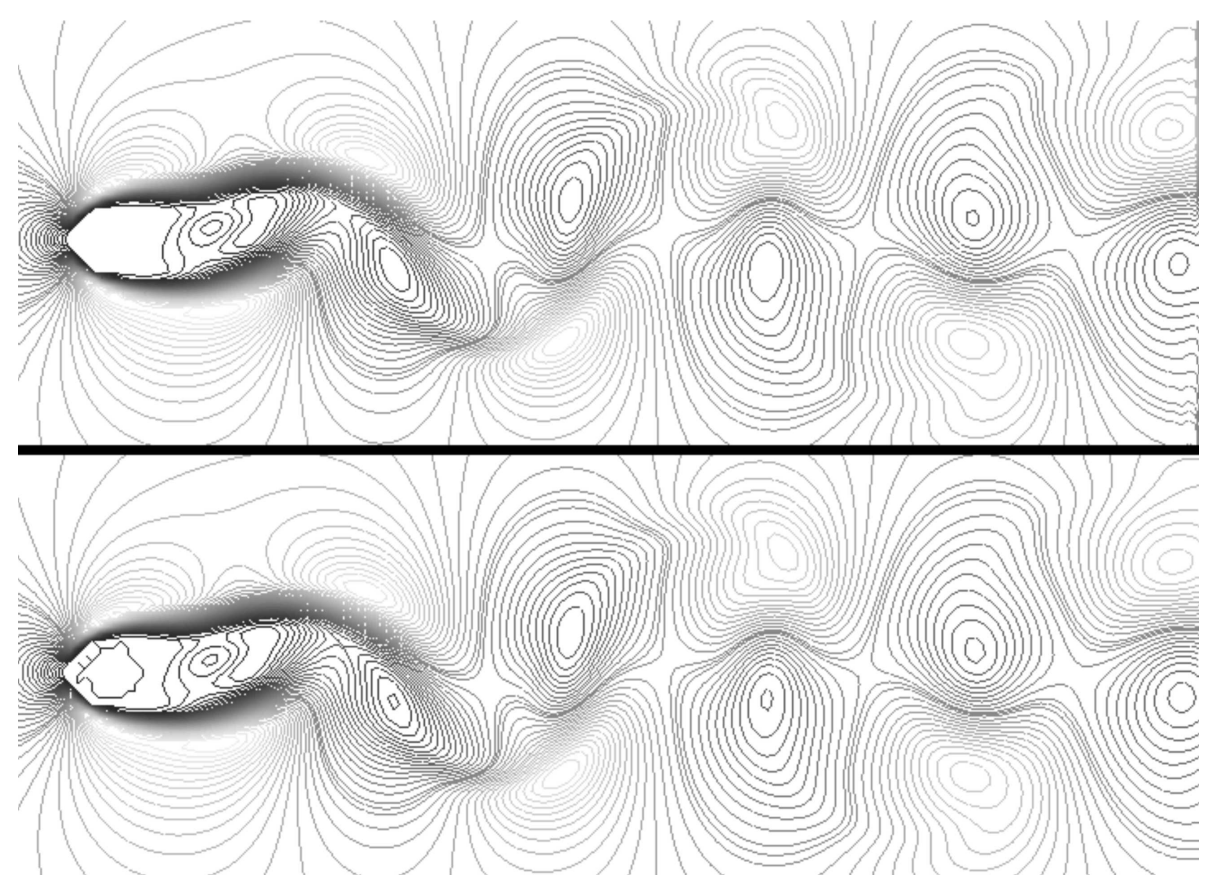

(a)

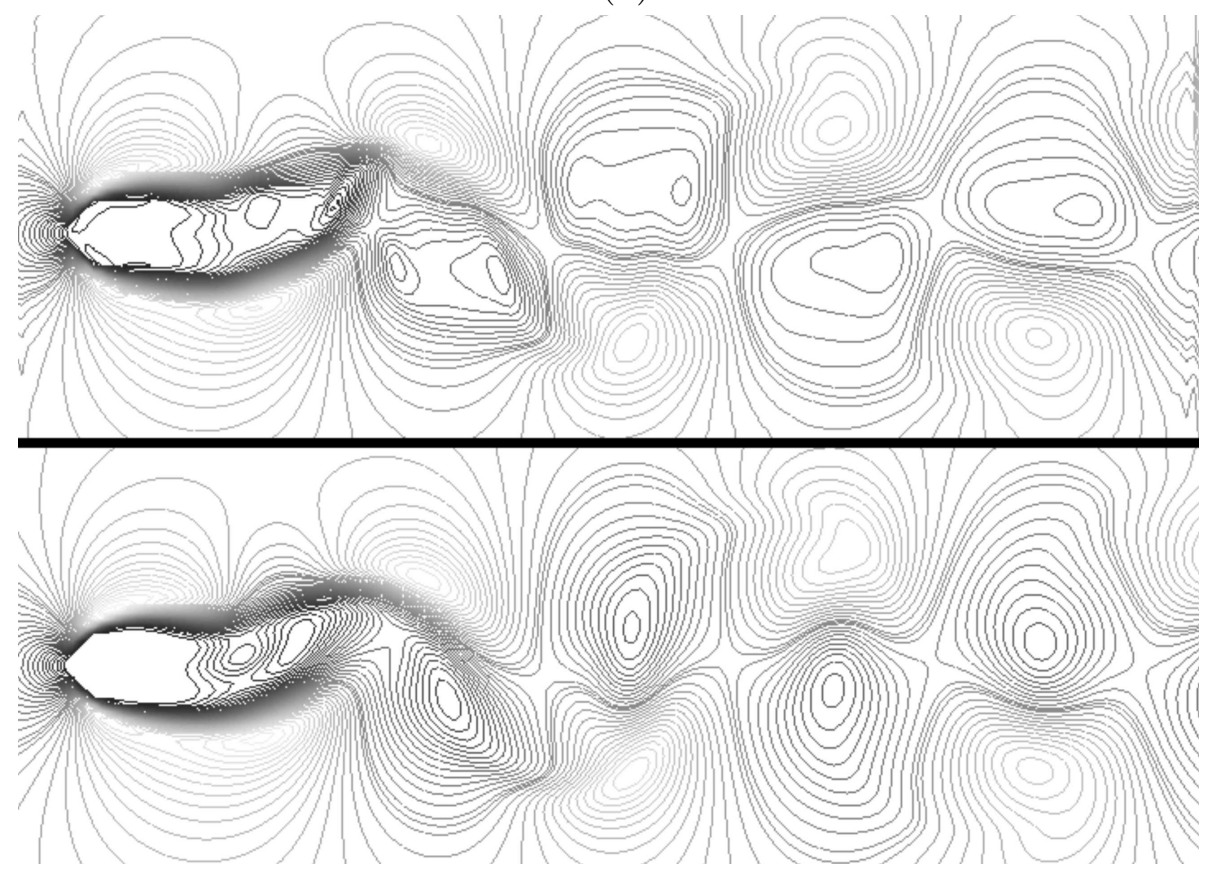

(b)

Figure 16: Isocontours (30 lines between the maximum and the minimum) of the reconstruction (upper line) and the simulation (lower line) for a) $t=0$ and for $t=1 / 8$ 


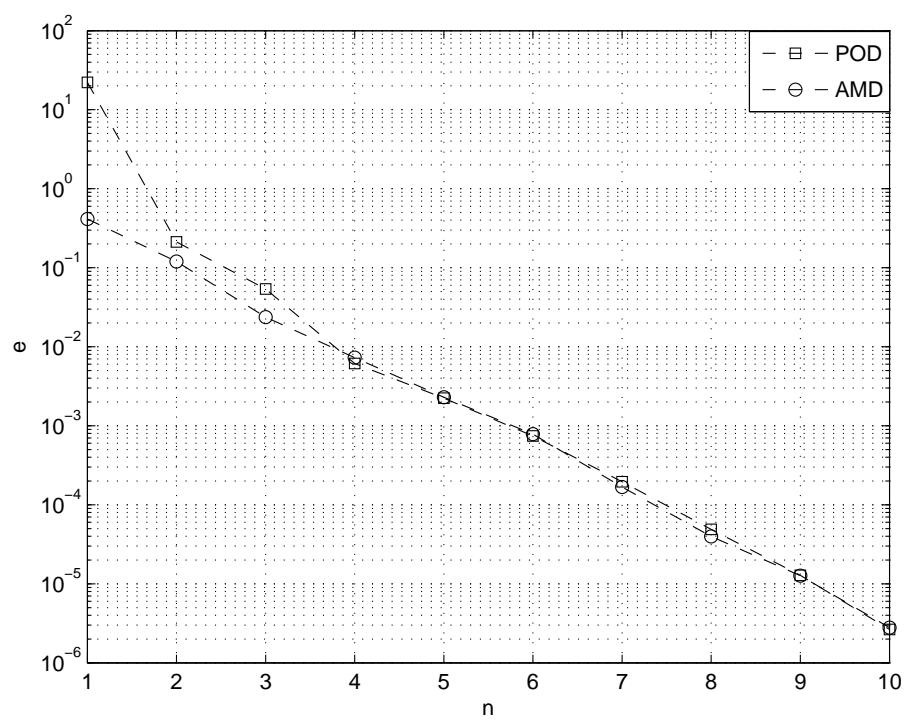

Figure 17: Spectrum of the autocorrelation matrix for advection mode decomposition and POD: eigenvalues in logarithmic scale.

to describe the periodic oscillation for all times.

\subsection{Hurricane Dean}

We consider the satellite images of the Caribbean see between the 17th and 22nd August 2007 showing the trajectory of hurricane Dean. The images are based on data from NOAA archive (http://www.aoc.noaa.gov/archive.htm) to get location and timeline of the storm. The images were generated by a NASA Goddard Space Center application (http://disc.sci.gsfc.nasa.gov/daac-bin/). Again, only 10 snapshots are considered. In this case, the density is simply defined as the normalized grey scale image. The time sampling was 6 hours, so that the time interval between the first snapshot and the last one is $T=54$ hours. In Fig. 18 three images are shown, at time $t=0, T / 2, T$. The resolution is $512 \times 256$.

Here, we concentrate on the possibility of using advection mode decomposition and POD to estimate a subsequent image not included in the initial database. The most energetic POD mode obtained from the 10 snapshots database is shown in Fig. 19.a): it is the average of the snapshots. The average position of the hurricane may be inferred from 

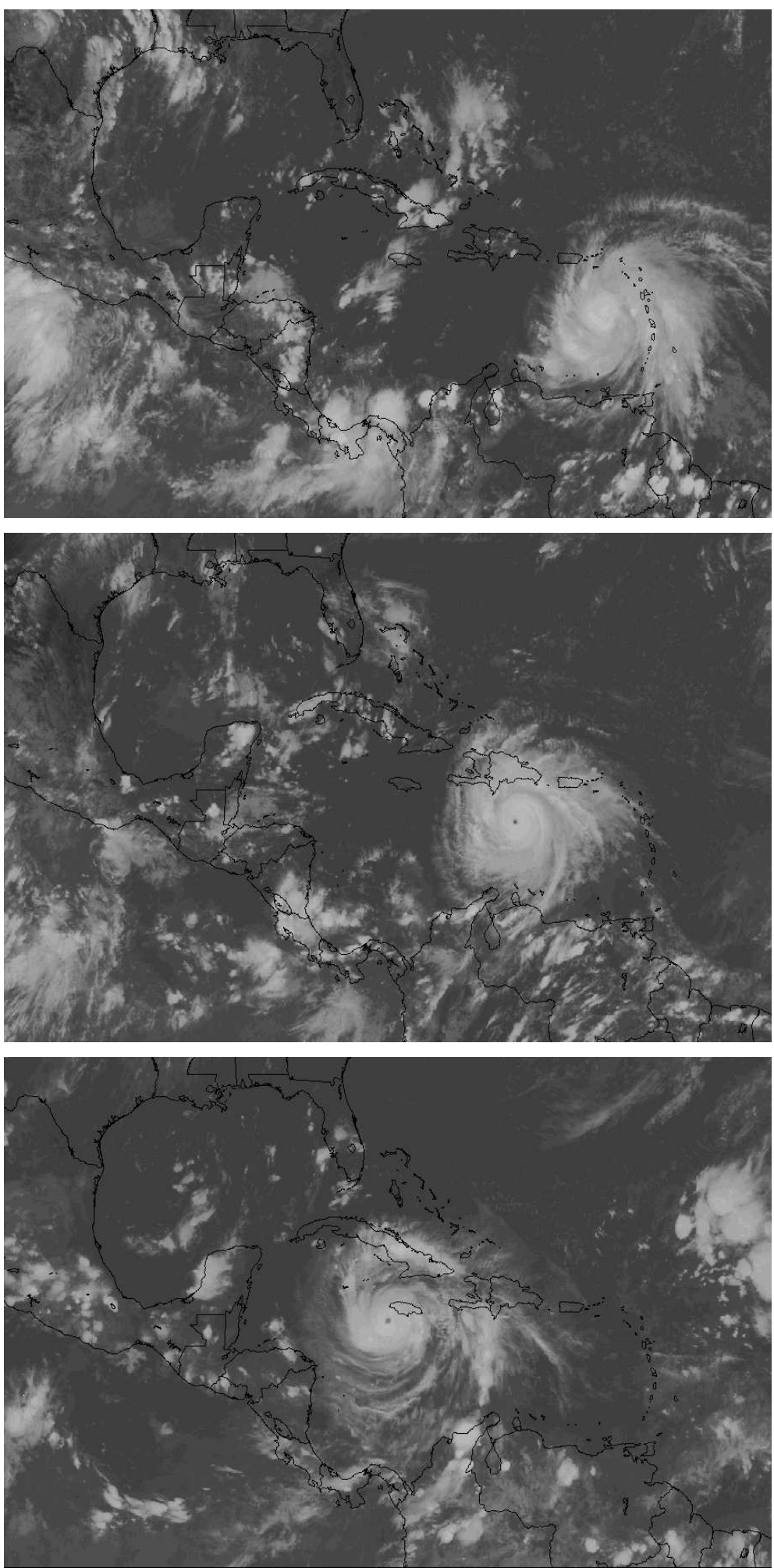

(a) top (b) middle (c) bottom

Figure 18: Images of the hurricane Dean at a )t=0, b) $t=27$ hours and c) 54 hours 
this picture. The other modes, see Fig. 19.b)-c), render the hurricane motion through global oscillating modes. No particular structure is visible in these modes. This is consistent with the fact that POD tends to the Fourier basis when it represents transported structures.

As for advection mode decomposition, a set of $N_{s}-1$ mapping is consider $\left(N_{s}=10\right.$ is the number of snapshots). This allows to map the snapshot $\varrho_{n}$ into $\varrho_{n+1}$. This set may be used to define, by numerical integration, a lagrangian coordinate for the system. Let $\phi_{n}$ be the mapping that transports $\varrho_{n}$ into $\varrho_{n}+1$. The following holds:

$$
X\left(\xi, t_{n}\right)=\int_{0}^{t_{n}} v d \tau=\int_{0}^{t_{n}} \nabla \phi d \tau=Q\left(\xi, \phi_{n}\right),
$$

where $X$ is the lagrangian coordinate and $Q$ is a quadrature formula. In this case a second order Adams-Bashforth scheme was used to integrate the mappings. Let us remark that $X$ is not the lagrangian coordinate of the physical system, but it is a mapping that allows to recover its state starting from the initial condition thanks to the Wasserstein map between each image. In Fig. 20 the lagrangian coordinate is represented at times $t=0, T / 2, T$. The deformation of the space is more intense around the hurricane (see for instance Fig. 20.b-c) ). This is due to the fact that its motion is more coherent than that of all the other structures. Optimal transport allows to highlight this feature in a straightforward manner.

We computed the transport modes and the first one is shown in Fig. ??. These modes represent potentials that suitably combined allow to map the first image of the sequence to all the others. With three transport modes, we can recover all the images with an error which is about $9 \%$ in norm $L^{2}$ norm of the gray scale.

We considered a subsequent hurricane image at time $T^{*}=60 \mathrm{~h}$ not included in the database to compute the POD modes and the advection modes. For POD representation, a simple problem of approximation is investigated, i.e., how accurately the new snapshot is represented using the POD modes computed using the database whose last snapshot is at $T=54 \mathrm{~h}$. The error of the reconstruction of this last image based on POD modes is about $16 \%$ using all the POD modes and does not vary significantly with the number of the modes used.

The approach based on Wasserstein distance allows to extrapolate the regularized lagrangian coordinate in a natural way. The discrete points (computed as explained above) 

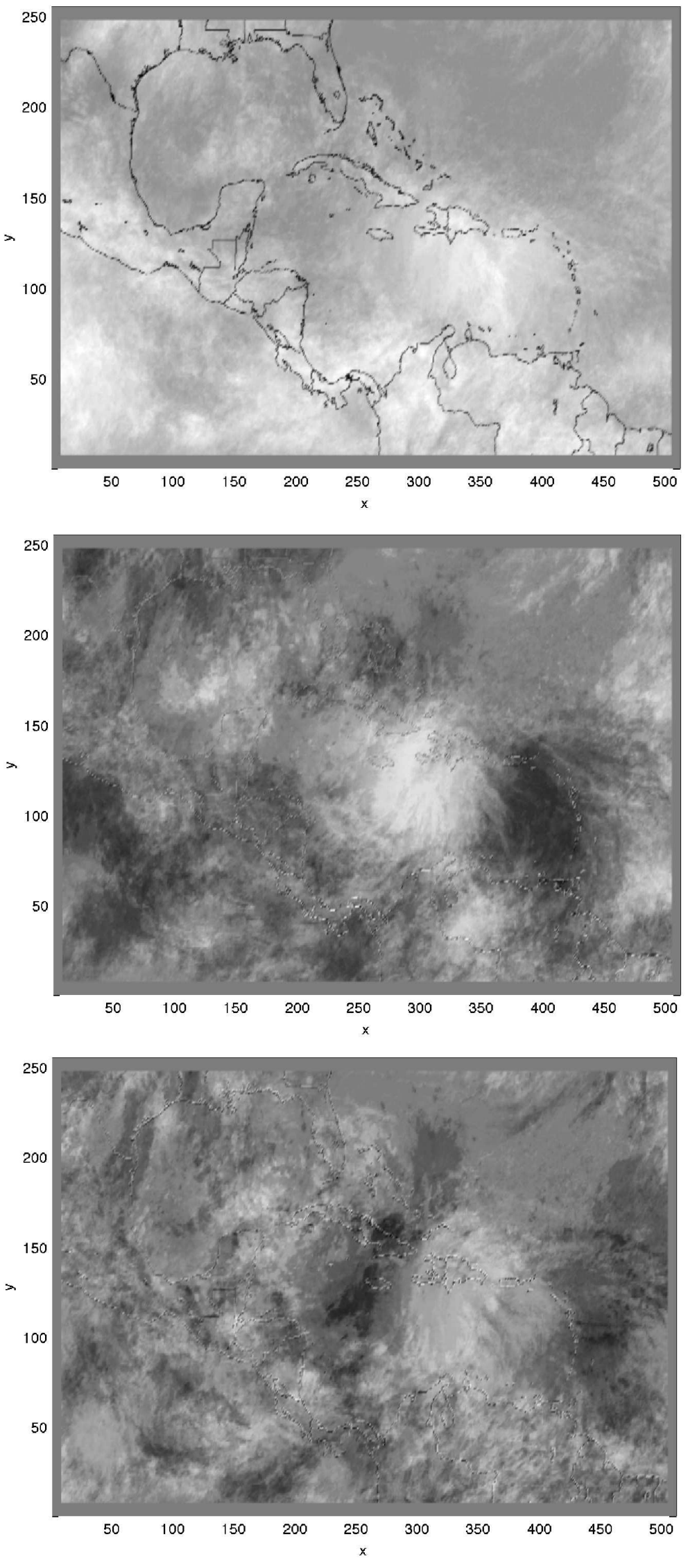

(a) top (b) middle (c) bottom

Figure 19: POD modes: a) first mode b) second mode c) third mode 28 

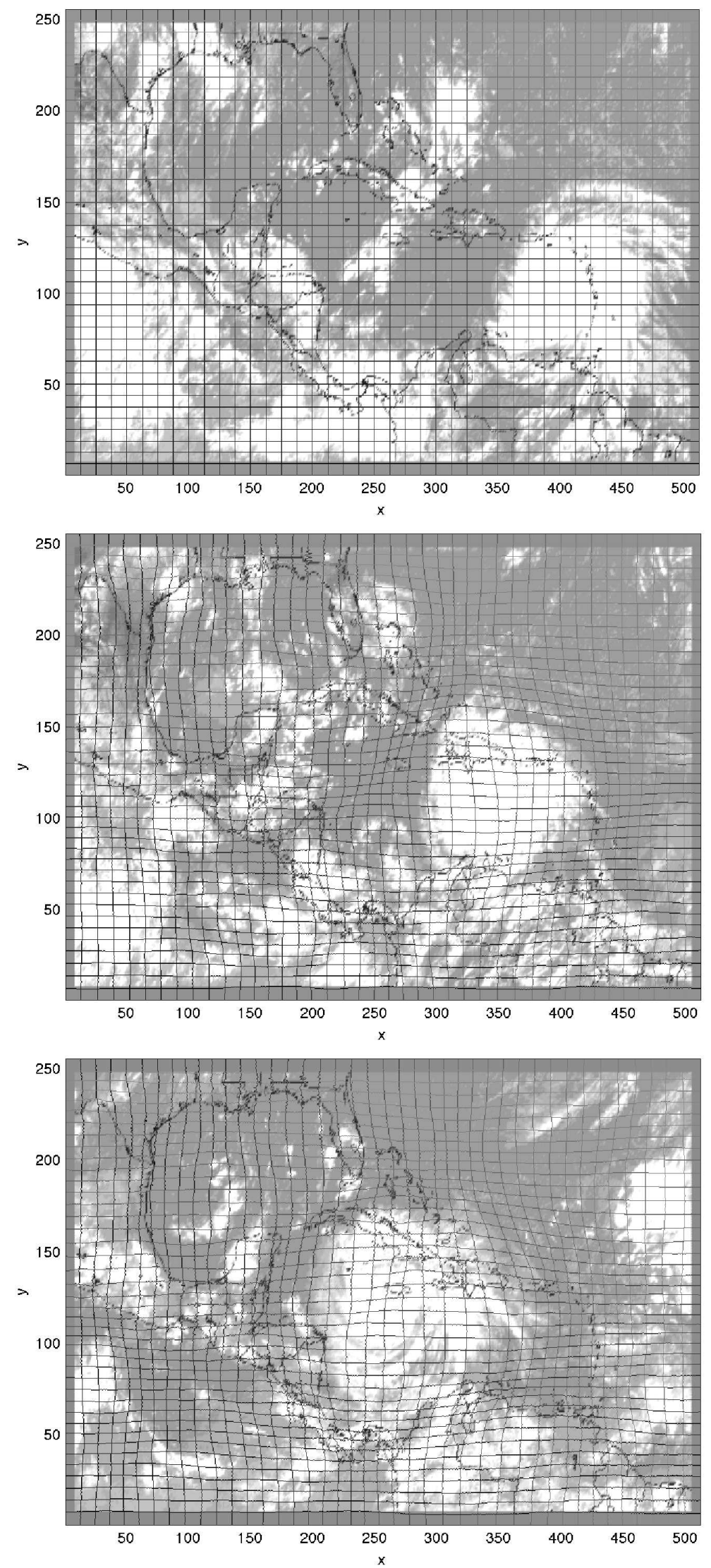

(a) top (b) middle (c) bottom

Figure 20: Lagrangian Coordinate at time a) $t=0$ b) $t=T / 2$ c) $t=T$ 


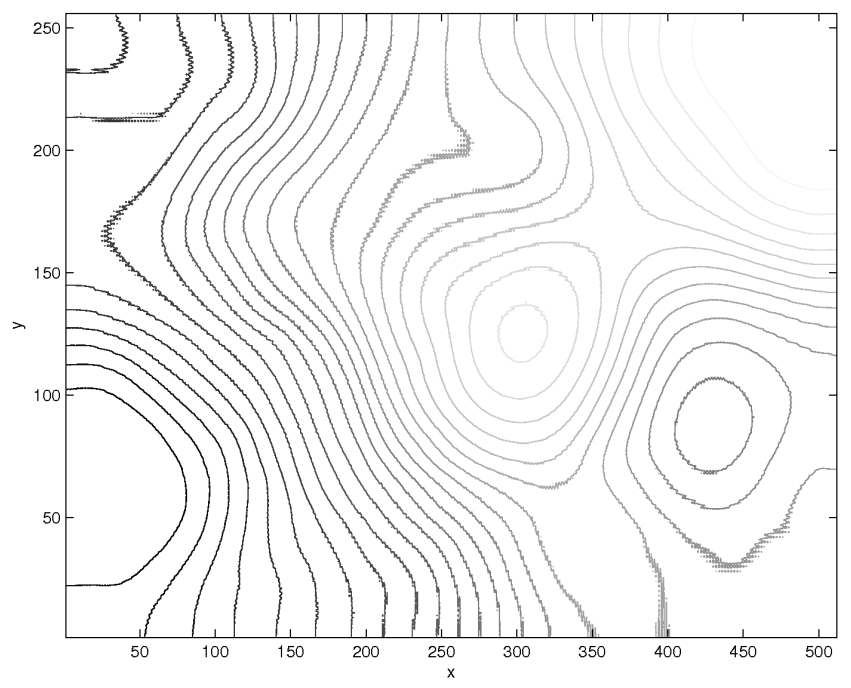

Figure 21: Isocontours of the first transport mode potential ( 25 values between maximum and minimum). Some isocontours are irregular because of numerical artifacts induced by noisy data in the corresponding regions.

$X\left(\xi, t_{n}\right), n=1, \ldots, 10$, are used to estimate $X\left(\xi, t_{n+1}\right)$ via a standard polynomial extrapolation. The error between the extrapolated lagrangian coordinate and that computed using of the novel datum at $T=60 \mathrm{~h}$ differs by 0.0108 in norm $L^{2}$. This means that the position of the hurricane is extrapolated with an error that is at most of $1 \%$. In terms of gray scale image reconstruction, the $L^{2}$ error of the whole field is about $9 \%$ and it is due essentially to the small random structures that are not well captured. The extrapolated image via advection modes is still better reconstructed compared to standard POD, for which we used the best possible estimate, i.e., the actual image.

\section{Conclusion}

In this paper we have proposed an advection based modal decomposition. It exploits the Wassestein distance to define a transport mode hierarchy that describes the main features of advection. Additional features are included by using a POD mode expansion of the residual in a reference domain. When dealing with systems in which transport is the leading phenomenon, this method provides an efficient and meaningful low-order representation. This has been shown by contrasting the results of this approach to standard 
POD analysis for different cases ranging from idealized vortex motion, to actual hurricane data.

\section{Appendix: Normalization of the embedding}

Let us suppose that the densities $\varrho_{i}$ are taken by uniformly sampling in time an optimal transport between $\varrho_{0}$ and $\varrho_{N_{s}-1}$. In this case, $X_{i}$, mapping $\varrho_{0}$ into $\varrho_{i}$ is written by interpolation (see [1]):

$$
X_{i}=\xi+i \Delta t \nabla_{\xi} \Phi(\xi)
$$

where $\Delta t$ is the sampling time, and $\Phi$ is a (almost everywhere) convex potential. In this particular case, all the densities are aligned on a one-dimensional subspace of the Wasserstein space, since they belong to the same optimal transport. Hence, we expect that only one eigenvalue of matrix $B$ is different from 0 .

The squared Wassertstein distance between the $i-t h$ and the $j-t h$ sample is:

$$
\mathcal{W}^{2}\left(\varrho_{i}, \varrho_{j}\right)=\int_{\Omega_{i}} \varrho_{i}(\eta)\left|X_{i j}(\eta)-\eta\right|^{2} d \eta
$$

where $X_{i j}(\eta)$ is the optimal mapping between $\varrho_{i}$ and $\varrho_{j}$. For these particular mappings the elements of the matrix have the form:

$$
\mathcal{D}_{i j}=\mathcal{W}^{2}\left(\varrho_{i}, \varrho_{j}\right)=\frac{C}{N_{s}^{2}}(i-j)^{2}
$$

where $\mathrm{C}$ is a constant, representing the squared Wasserstein distance (i.e. twice the kinetic energy) of the unique mapping linking all the snapshots. The time at which the last snapshot is taken is taken to be $T=1$.

Let us consider the matrix $\tilde{\mathcal{D}}=(i-j)^{2}, \tilde{\mathcal{D}} \in \mathbb{R}^{n \times n}$ and prove that the associated embedding matrix $\mathrm{B}$ has only one zero eigenvalue and that this value is $\lambda=\frac{n(n+1)(n-1)}{12}$.

The elements of $B$ are computed using two standard results in finite series:

$$
\sum_{j}^{n} j=\frac{n(n+1)}{2}, \quad \sum_{j}^{n} j^{2}=\frac{n(n+1)(2 n+1)}{6} .
$$

By performing all the matrix vector products and exploiting the projector properties we have:

$$
-(2 \mathcal{B})_{i j}=(n+1)(i+j)-2 i j-\frac{(n+1)^{2}}{2} \Rightarrow \mathcal{B}_{i j}=\frac{(n+1)^{2}}{4}+i j-\frac{(n+1)}{2}(i+j)
$$


Let $k=\frac{n+1}{2}$. The expression for the entries of $B$ can be recast as follows:

$$
\mathcal{B}_{i j}=k^{2}-k(i+j)+i j \Rightarrow \mathcal{B}_{i j}=(k-i)(k-j) .
$$

The relation written above states that $B$ is the tensor product of a unique vector, whose components are $y_{i}=(k-i)$. This is sufficient to prove the first point. Let us now explicitly compute the only non-zero eigenvalue $\lambda$. Again, the results on finite series are used, leading to:

$$
\lambda=\sum_{i}^{n}(k-i)^{2}=\frac{n(n+1)(n-1)}{12} .
$$

The normalization condition for the generic $B \in \mathbb{R}^{N_{s} \times N_{s}}$ is then:

$$
\bar{B}=\frac{B}{\mathcal{N}}=-\frac{6 N_{s}}{N_{s}^{2}-1} J D J
$$

\section{References}

[1] J-D. Benamou and Y. Brenier. A computational fluid mechanics solution to the Monge-Kantorovich mass transfer problem. Numerische Matematik, 84:375-393, 2000 .

[2] L.C. Evans. Partial differential equations and Monge-Kantorovich mass transfer. Technical report, University of California, Berkley, 1997.

[3] A. Iollo and D. Lombardi. A lagrangian scheme for the solution of the optimal mass transfer problem. Journal of Computational Physics, 230:3430-3442, 2011.

[4] Muskulus M. and Verduyn-Lunel S. Wasserstein distances in the analysis of time series anddynamical systems. Physica D, 240:45-58, 2011.

[5] V.V. Meleshko and M.Y. Konstantinov. Vortex Dynamics and chaotic phenomena. World Scientific Publishing Company, 1st edition, 2002.

[6] I. Mezić. Spectral properties of dynamical systems, model reduction and decompositions. Nonlinear Dyn., 41:309-325, 2005.

[7] Holmes P., Lumley J.L., and Berkooz G. Turbulence, coherent structures, dynamical systems and symmetry. Cambridge University Press, 1st edition, 1996. 
[8] Schmid P. Dynamic mode decomposition of numerical and experimental data. $J$. Fluid. Mech., 656:5-28, 2010.

[9] C.W. Rowley, I. Mezić, Bagheri S., Schlatter P., and Henningson D.S. Spectral analysis of nonlinear flows. J. Fluid. Mech., 641:115-127, 2009.

[10] L. Sirovich. Low dimensional description of complicated phenomena. Contemporary Mathematics, 99:277-305, 1989.

[11] C. Villani. Topics in optimal transportation. American Mathematical Society, 1st edition, 2003.

[12] C. Villani. Optimal Transport, old and new. Springer-Verlag, 1st edition, 2009. 